Full Length Article

\title{
A 3D coupled model of organic matter and inorganic matrix for calculating the permeability of shale
}

\author{
Gaohui Cao ${ }^{\mathrm{a}, \mathrm{b}}$, Mian Lin ${ }^{\mathrm{a}, \mathrm{b}, *}$, Wenbin Jiang ${ }^{\mathrm{a}}$, Haishan $\mathrm{Li}^{\mathrm{a}}$, Zhixing $\mathrm{Yi}^{\mathrm{a}}$, Chenjie Wu ${ }^{\mathrm{a}, \mathrm{b}}$ \\ a Institute of Mechanics, Chinese Academy of Sciences, Beijing 100190, China \\ ${ }^{\mathrm{b}}$ School of Engineering Science, University of Chinese Academy of Sciences, Beijing 100049, China
}

\section{H I G H L I G H T S}

- A new 3D model coupling organic kerogen and inorganic matrix was developed.

- The sensitivity analysis on the apparent permeability of shale matrix were operated.

- Ignoring the contribution of kerogen to the permeability of shale will bring great error in most cases.

- When the TOC is less than $11 \%$ and the pressure is greater than $0.1 \mathrm{Mpa}$, the effect of kerogen distribution is negligible.

\section{A R T I C L E I N F O}

\section{Article history:}

Received 5 January 2017

Received in revised form 20 April 2017

Accepted 12 May 2017

Available online 22 May 2017

\section{Keywords:}

Multiscale

3D coupled model

Mortar

Apparent permeability

Shale gas

\begin{abstract}
A B S T R A C T
Microstructure-based quantitative computation of the shale permeability is challenging due to the presence of organic matter with nanoporous features. In this study, a new three-dimensional (3D) coupled model is proposed to investigate the micro-scale permeability of organic-rich-shale matrix. The coupled model consists of two subdomains, organic matter and inorganic matrix. They are described by the pore network model (PNM) and continuum model to capture the non-Darcy and Darcy flow, respectively, and the gas flow in the two subdomains are coupled by finite element mortars (Mortar). The convergence error, grid discretization and parallel scheme are also investigated to get the optimal computing parameters for this model. Then, the effects of the total organic content (TOC), kerogen distribution and poresize distribution on the apparent permeability (AP) of shale matrix are studied using the coupled model with computer-generated PNMs. And on the basis of FIB-SEM images, a Longmaxi shale sample from the Sichuan Basin, China is introduced to build a real shale model. Results show that AP is more sensitive to TOC and pore-size distribution than kerogen distribution. Additionally, in order to analyze the necessity of 3D model, a comparison of 3D and two-dimensional (2D) model is made and the error of 2D model is pointed out. The 3D couple model affords a foundation for further upscaling of shale permeability to REV scale and reservoir scale.
\end{abstract}

(c) 2017 Elsevier Ltd. All rights reserved.

\section{Introduction}

Shale gas, generated by the thermal evolution of organic matters (kerogen) and constrained within the ultra-tight shale, has attracted significant attentions to meet world's increasing energy demand. In shale reservoirs, the gas flow through very small pores (in nanoscale) to micro-fracture networks [1], and using conventional approaches (such as Darcy's law) in the whole region is chal-

* Corresponding author at: Institute of Mechanics, Chinese Academy of Sciences, Beijing 100190, China.

E-mail address: linmian@imech.ac.cn (M. Lin). lenging [2-4]. The permeability varies with the pressure, temperature, porosity and pore size, as well as the size of shale rocks because flow regime varies in different scales $[5,6]$.

It is obvious, for example, from the focused ion beam scanning electron microscope (FIB/SEM) images of a Longmaxi shale sample (Fig. 1), that there are two kinds of subdomains: kerogen with plenty of nanopores and inorganic matrix with micro intergranular pores and micro-fractures [7-9]. And the nanopores of kerogen form pore networks with good connectivity, while in inorganic matrix the pores or fractures can hardly be seen. The isolated kerogen blocks are dispersed in shale. Recently, numerous researchers 


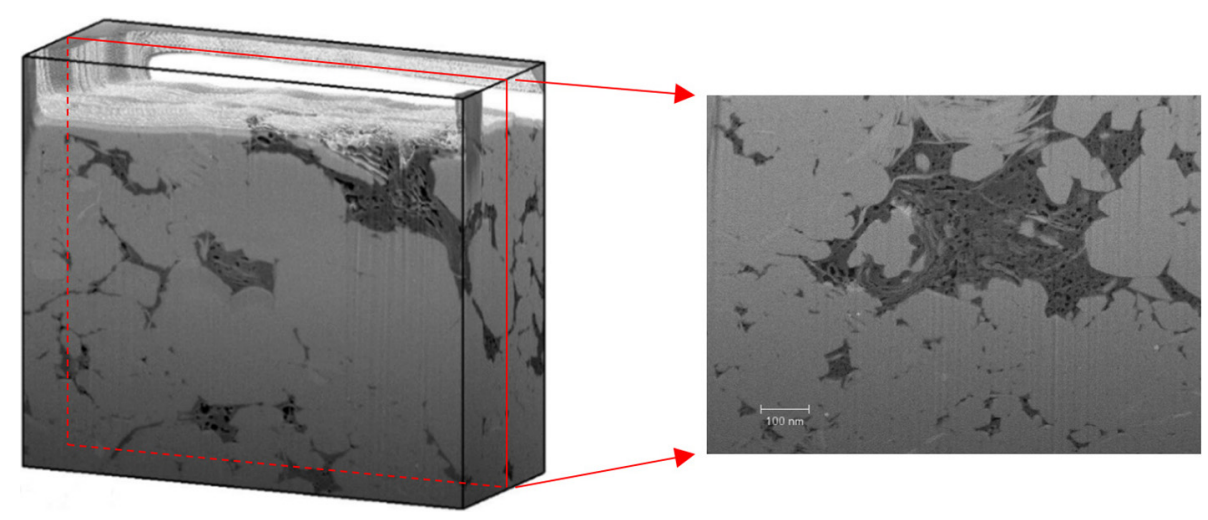

Fig. 1. FIB/SEM images of a Longmaxi Shale sample. Light grey indicated the inorganic matrix, dark grey indicated organic matter; and deep black indicated nanopores.

have noticed the big differences between kerogen and inorganic matrix in shale and treat them separately [10-17].

Akkutlu and Fathi [10] used lattice Boltzmann method (LBM) to simulate gas dynamics in organic matter and found that Darcy's law is applicable when organic pores are extremely large $(>100 \mathrm{~nm})$. Later, they [11] proposed a dual-porosity model to separate kerogen from inorganic matrix and pointed out that gas transport is dominated by Darcy flow in inorganic matrix while by non-Darcy flow in kerogen. They concluded that the permeability of shale is primarily relevant to the relatively large pore and fractures in inorganic matrix, but the molecular behaviors (diffusion transport and nonlinear adsorption) relevant to the pores in kerogen. In 2015, Akkutlu [12] adopted multiscale asymptotic analysis method to solve a one-dimensional dual-porosity continuum model and concluded that both the gas in-place and gas production rate depend on the content of kerogen in shale. Later, Akkutlu [13] employed the generalized multiscale finite element method (GMsFEM) to add the influence of fractures and extended the model to 2D. Based on Akkutlu's work, in 2016, Alexey [14] divided shale into kerogen and inorganic matrix and solved both the continuum subdomains by nonlinear parabolic equation, then utilized numerical homogenization technique through local problems to calculate macro parameters. However, it is not convenient to include the influence of local heterogeneity caused by microand nano- pores when both the organic and inorganic matter are represented by continuums.

Song et al. [15] performed a study on the influence of pore pressure on the apparent permeability. They showed that pore pressure will affect the permeability of organic matter but has no effect on the permeability of inorganic matter. Additionally, the study assumed that organic matter and inorganic matter are isotropic, and discussed the apparent permeability with the mean pore size. A real shale is of intensely strong heterogeneity and anisotropy, calculating the permeability by a single pore size will bring errors and the pore-size distribution should be taken into consideration. Naraghi and Javadpour [16] put the pore-size distribution measured by the 3D experiment into a 2D model by stochastic classification method based on statistics, and applied ExpectationMaximization algorithm (EM) to separate two distributions of pores in organic matter and inorganic matrix, then discussed the impact of total organic content (TOC) and the pore-size distribution on AP. After that, Wang et al. [17] employed EM algorithm to divide the pore size distribution and applied generalized lattice Boltzmann model (GLBM) to solve a 2D problem to analyze the effect of organic and inorganic matter distribution, TOC and surface diffusion on permeability. It is found that the distribution of organic and inorganic matter has a negligible influence on appar- ent permeability, but TOC and surface diffusion are of significant importance. However, Chen [18] supposed that the gas exchange within organic matter is a slow process, so gas transport occurs merely in the inorganic matrix and the organic matter has no effect on gas permeability, in addition, the mass exchange rate of the kerogen and inorganic matrix is linearly proportional to the free gas pressure difference between them. Although those models separate kerogen from inorganic matrix, 2D model is widely used without considering the 3D heterogeneity and anisotropy.

In recent years, several approaches are proposed for investigating multiscale effects. Scheibe, Tartovosky, Battiato [19] present a general formulation of an iterative hybrid numerical method that links the pore and continuum scales. Sheng and Thompson [20] present a concurrent coupling method of pore-scale and continuum-scale. Chen and Prodanovic [21] utilized a random walk algorithm to link the restricted diffusion coefficient to the pore scale characteristics of shale gas media. Tomlin [22] describes an adaptive gridding models for pollution transport and reaction using a layered and fully adaptive 3-D tetrahedral approach. However, those coupling strategies had limitations in terms of flexibility and efficiency. The limitations were avoided in a later work through the introduction of Mortar [23]. The advantages of Mortar are that it allows for different physics, scales, and models in various parts of the domain and is easily parallelizable. It would be of great value if different parts of the domain could be modeled through different methods which are appropriate to the local transport conditions and level of accuracies.

Kerogens play an important role in the storage and transport of shale gas, but most of them are isolated from one another. However, gas flow in a real shale is a coupled system of kerogen and inorganic matrix. Either the continuum models or the statistic models can hardly describe the shale characteristics integrally. Thus, in the study, we propose a 3D coupled model. It is a model where a number of kerogens disperse randomly in inorganic matrix. Kerogen is represented by pore network model (PNM) owing to its pore size is of nanometer magnitude and its gas transport covers viscous flow, slip flow and Knudsen diffusion. On the other hand, inorganic matrix is regarded as an isotropic porous medium where gas flow occurs primarily in micro channels and fractures. It is assumed that gas flow in inorganic matrix satisfies Darcy's law and finite volume method (FVM) is utilized to represent it. Then, Mortar method is employed to couple kerogen and inorganic matrix models together by enforcing the continuity of pressures and fluxes at the shared boundary interfaces. Finally, the coupled model is solved by parallel computing.

This paper is organized as follows. Section 2 presents the principles, derivation, validation and computational efficiency of the 
coupled model. In Section 3, the sensitivities of the apparent gas permeability to TOC, kerogen distribution, pore-size distribution and space dimension are analyzed based on the coupled model with the regular-topology PNMs generated by computer. Moreover, the real-shale PNMs extracted from FIB/SEM images are also analyzed here. Finally, the summary and conclusions of this work are drawn in Section 4.

\section{Coupled model development}

From FIB/SEM images, it can be seen that a series of organic matters (kerogen) are surrounded by inorganic matrix. Inside the kerogen, there exist many developed pore networks with good conductivity. Therefore, a coupled model in which kerogen disperses within inorganic matrix is established in this study, as shown in Fig. 2.

\subsection{Gas flow model for nanoporous kerogen}

PNM is adopted to describe the porous space in kerogen. It is a pore-scale technique in which the porous media is approximated as an interconnected network of pores and throats. In this work, both computer-generated networks and real-shale networks using FIB/SEM will be used. For computer-generated networks, it is usually assumed that pore and throat sizes are spatially uncorrelated in the regular-topological networks, but previous studies have found that the size relationship between the adjacent pores and throats has certain effects on the permeability of the networks [24]. In order to guarantee the spatial correlation between pores and throats, a PNM based on the work of Blunt et al. [25] in which large pores prefer to be connected with large throats is employed in this study. For real-shale networks, the $A B$ (axis \& ball) algorithm [26] is employed to extract the PNMs from FIB/SEM images.

The general approach to PNM is to impose a mass conservation equation at each pore in the network. For compressible steady flows, the conservation equation for pore $i$ is described as

$\sum_{j} g_{i j}\left(p_{i}-p_{j}\right)=0$

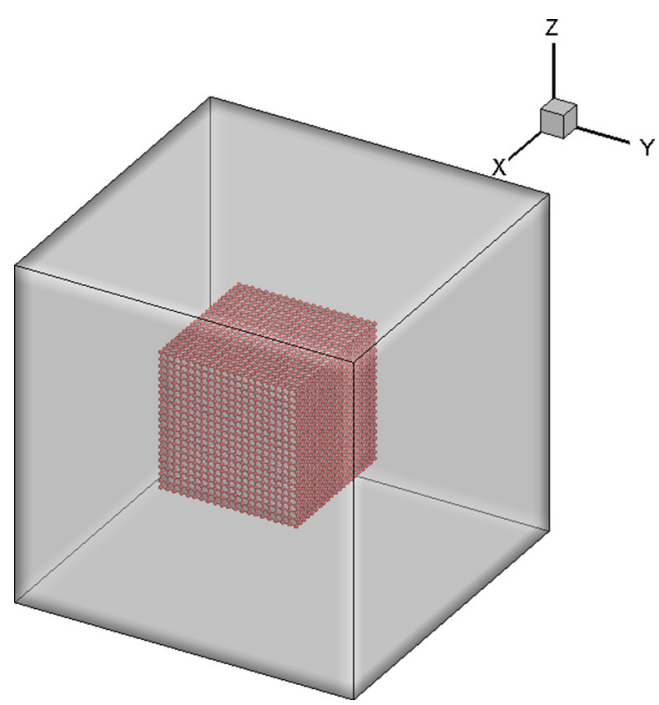

Fig. 2. Coupled model (the inner porous kerogen is represented by PNM and the surrounding inorganic matrix is represented by FVM, and their interfaces are coupled by Mortar). where $p_{i}$ and $p_{j}$ are the pressure of pore $i$ and its adjacent pore $j . g_{i j}$ is the mass flow conductivity, which satisfies:

$g_{i j}=\frac{K_{a p p} \pi r_{i j}^{2}}{\mu l_{i j}} \rho_{a v g}$

where $r_{i j}$ is the radius of the throat which connects pore $i$ and pore $j$; $l_{i j}$ is the throat length; $\mu$ is gas viscosity; $\rho_{a v g}$ is gas density, calculated by $\rho_{\text {avg }}=\frac{p_{\text {avg }} M}{Z R T}, p_{\text {avg }}$ is the average pressure in the throat; $M$ is molecular molar mass of methane; $Z$ is compressibility factor; $R$ is gas constant; $T$ is absolute temperature; $K_{a p p}$ is the apparent permeability of the throat calculated by Javadpour's formula [27]:

$$
\begin{aligned}
K_{a p p}= & \frac{2 r_{i j} \mu M}{3 R T \rho_{a v g}}\left(\frac{8 R T}{\pi M}\right)^{0.5} \\
& +\left\{1+\left(\frac{8 \pi R T}{M}\right)^{0.5} \frac{\mu}{p_{a v g} r_{i j}}\left(\frac{2}{\alpha}-1\right)\right\} \frac{r_{i j}^{2}}{8}
\end{aligned}
$$

where $\alpha$ is the tangential momentum accommodation coefficient (TMAC). In this work, the temperature is assumed as a constant and the pressure difference between the inlet and outlet is subtle, $\mu Z$ is taken as a constant. Substituting $g_{i j}$ into Eq. (1), using the square pressure form, one obtains:

$\sum_{j} \frac{K_{a p p} \pi r_{i j}^{2}}{l_{i j}} \frac{M}{2 \mu Z R T}\left(p_{\mathrm{i}}^{2}-p_{j}^{2}\right)=0$

Regarding the square pressure $\tilde{p}=p^{2}$ as the unknown, the Eq. (4) is simplified as:

$\sum_{j} \frac{K_{a p p} \pi r_{i j}^{2}}{l_{i j}} \lambda\left(\tilde{p}_{i}-\tilde{p}_{j}\right)=0, \lambda=\frac{M}{2 \mu Z R T}$

\subsection{Gas flow model for inorganic matrix}

Considering gas flow in inorganic matrix is dominated by Darcy flow, it is modeled by FVM. For steady flows, the conservation equation for grid $i$ is simply

$\sum_{j} T_{i j}\left(p_{i}-p_{j}\right)=0$

For compressible fluids, mass flow conductivity $T_{i j}$ can be written as:

$T_{i j}=\frac{K_{i o m} A_{i j}}{\mu d_{i j}} \rho_{a v g}$

where $p_{i}$ and $p_{j}$ are the pressure of grid $i$ and its adjacent grid $j ; K_{\text {iom }}$ is the Darcy permeability of inorganic matrix; $A_{i j}$ is the area of the grid interface; $d_{i j}$ is the distance between the centers of grid $i$ and grid $j ; \rho_{\text {avg }}$ is gas density, calculated by $\rho_{\text {avg }}=\frac{p_{a v y} M}{Z R T} ; p_{a v g}$ is the average pressure. Taking $\mu Z$ as a constant, the Eq. (6) can also be changed into the form of square pressure:

$\sum_{j} \frac{K_{i o m} A_{i j}}{d_{i j}} \frac{M}{2 \mu Z R T}\left(p_{\mathrm{i}}^{2}-p_{j}^{2}\right)=0$

Regarding the square pressure as the unknown, the Eq. (8) can be simplified as:

$\sum_{j} \frac{K_{i o m} A_{i j}}{d_{i j}} \lambda\left(\tilde{p}_{i}-\tilde{p}_{j}\right)=0, \lambda=\frac{M}{2 \mu Z R T}$

In the inorganic matrix, permeability is served as a constant: $K_{\text {iom }}=$ const 


\subsection{Mortar coupling}

In this study, Mortar method is employed to connect kerogen and inorganic matrix. Mortar is proposed by Bernardi, Maday and Patera [28] to solve the Possion problem at first. It decomposes the solution domain into several subdomains, then discretely solves the subdomains with the most suitable method according to their characteristics using a convenient parallel computing. Balhoff et al. [23,29] extended Mortar for the first time to pore-scale models (such as coupling PNM to PNM and PNM to continuum). This study follows the idea of Balhoff and uses Mortar to realize the coupling of PNM and continuum which represent kerogen and inorganic matrix, respectively (Fig. 3). In inorganic matrix, FVM is chosen in order to ensure the local conservation of fluxes.

Mortar method can be referred from Balhoff [23] and it requests that the coupled interface must satisfy: (1) The pressure of the left and right subdomains of the interface is continuous. This rule is guaranteed by the property that the pressure of the boundary nodes of the subdomains is obtained based on their position in Mortar element. (2) The fluxes should be matched at each interface in a weak sense (explanation comes next).

The pressure field of Mortar space is a linear combination of basic functions as:

$P(x, y)=\sum_{j} \alpha_{j} \phi_{j}(x, y)$

where $P(x, y)$ is the pressure field, $\phi_{j}(x, y)$ is the basic functions of the $j$ th Mortar node, $\alpha_{j}$ is the coefficients of basic functions. The basic functions can vary hierarchically from constant, linear, quadratic, etc. order functions. Here, the quadrilateral element and the piecewise continuous bi-linear basis functions are applied. The solution is obtained by determining the coefficients ( $\alpha_{j}$ 's) of basic functions that maintains the weak match of fluxes in the interfaces. The resulting flux across each entire interface is matched in such a way that total mass is conserved. In order to make the fluxes of the left and right subdomains adjacent to the interfaces is integrally equal (that is, the weak match), one obtains:

$F_{j}\left(\alpha_{1}, \alpha_{2}, \ldots, \alpha_{D O F}\right)=\int q_{L} \phi_{j}-q_{R} \phi_{j}=0,(j=1,2, \ldots, D O F)$

where $F_{j}$ is the jump in fluxes across the interface, $q_{L}$ and $q_{R}$ are the fluxes in the left and right side of the interfaces, respectively. DOF $=$ degrees of freedom $=$ basis function number $=$ Mortar node number. The mathematical approach for Mortar method is solving the nonlinear equations Eq. (11) by Newton-Raphson iterative methods, a summary of the procedure is shown as follows:

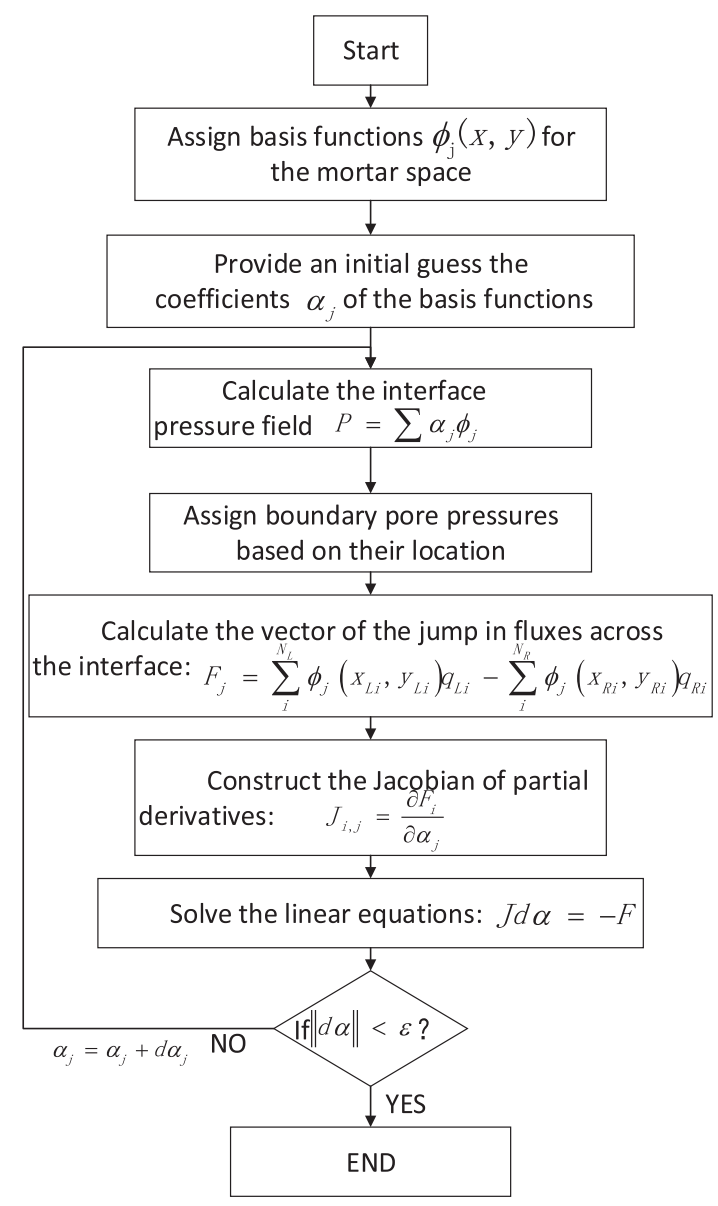

where $\left(x_{L i}, y_{L i}\right)$ and $\left(x_{R i}, y_{R i}\right)$ are the coordinates of the FVM nodes or PNM pores in the left and right side of the interface, $N_{L}$ and $N_{R}$ are the number of those nodes or pores; $J$ is Jacobian matrix; $\varepsilon$ is the calculation accuracy.

\subsection{Apparent permeability of the coupled model}

A Diriclet boundary condition is applied to the coupled model with a constant inlet pressure $p_{i n}$ and outlet pressure $p_{\text {out }}$. And the definition of the apparent permeability of the coupled model $K_{c p}$ is given by:

$K_{c p}=\frac{q_{m}}{A_{c p}} \frac{2 \mu Z R T}{M} \frac{L_{c p}}{\left(\tilde{p}_{\text {in }}-\tilde{p}_{\text {out }}\right)}$

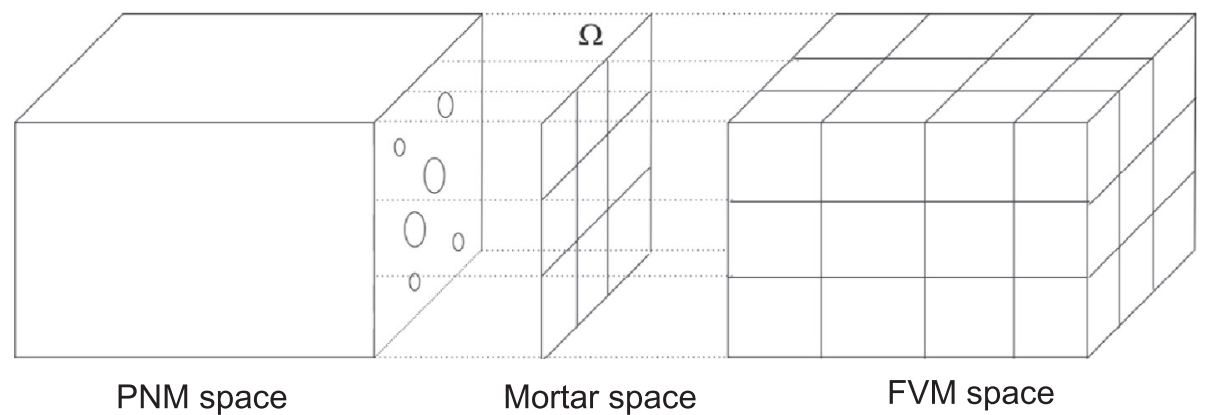

Fig. 3. Mortar coupling of PNM and FVM. 
where $A_{c p}$ is the sectional area of the coupled model, $L_{c p}$ is the length along the flow direction; $\tilde{p}=p^{2} ; q_{m}$ is the total flow mass aross the boundary expressed by:

$q_{m}=\sum_{i \in \partial \Omega} \sum_{j} \frac{K_{i o m} S_{i j}}{d_{i j}} \frac{M}{2 \mu Z R T}\left(\tilde{p}_{i}-\tilde{p}_{j}\right)$

where $\partial \Omega$ is the boundary of the coupled model. Substituting Eq. (13) to Eq. (12), the apparent permeability can be rewritten as:

$K_{c p}=\frac{1}{A_{c p}} \frac{L_{c p}}{\left(\tilde{p}_{\text {in }}-\tilde{p}_{\text {out }}\right)} \sum_{i \in \partial \Omega} \sum_{j} \frac{K_{\text {iom }} S_{i j}}{d_{i j}}\left(\tilde{p}_{i}-\tilde{p}_{j}\right)$

\subsection{Model validation}

In this section, three kinds of conditions are found (Fig. 4) to validate our model and codes. The inside of these conditions is medium 1 with a constant permeability and the external is medium 2 with a permeability which varies with pressure. Medium 1 represents inorganic matrix while medium 2 represents kerogen. Here the PNM (represents kerogen) is homogeneous and isotropous and has a regular cubic topology. It is easy to get the upscaled permeability of this PNM and utilize a continuum media to replace it to get an equivalent result as condition 2\&3 (FVM-Mortar-FVM and Global FVM). The three conditions are enumerated in Table 1, and their Apparent Permeability Function (APF) curves are shown in Fig. 5.

The APF curves of the three conditions are well matched (Fig. 5). It proves that Mortar method is feasible to deal with the FVM-PNM and FVM-FVM interface. In addition, our program codes have been validated.

\subsection{Discussion of computational efficiency}

\subsubsection{Solution accuracy of different spaces}

In this section, the solution accuracy of different spaces is studied. There are three spaces in the calculation: PNM space, FVM space and Mortar space. In Mortar space, Newton-Raphson iteration converges quickly, its convergence error achieves 1e-6 through only 2 iteration steps. However, the accuracy and efficiency still should be considered in the solution of the steady flow in PNM and FVM, as shown in Table 2.

The accuracies of the two subdomains (PNM and FVM space) should match with the accuracy of Mortar space to improve the efficiency. The efficiency is maximized when the accuracy of subdomains is 1-2 orders of magnitude higher than that of Mortar space. In this study, the convergence error of the NewtonRaphson iteration is set to $1 \mathrm{e}-6$ and the steady convergence errors of PNM and FVM are set to $1 \mathrm{e}-8$. In such a condition, the deviation
Table 1

Properties of the three conditions.

\begin{tabular}{clll}
\hline & Medium 1 & Medium 2 & Interface \\
\hline PNM-Mortar-FVM & $\begin{array}{l}\text { Continuum } \\
(k=\text { const })\end{array}$ & PNM $(k=f(p))$ & $\begin{array}{l}\text { Mortar coupling } \\
\text { interface }\end{array}$ \\
FVM-Mortar-FVM & $\begin{array}{l}\text { Continuum } \\
(k=\text { const })\end{array}$ & $\begin{array}{l}\text { Continuum } \\
(k=f(p))\end{array}$ & $\begin{array}{l}\text { Mortar coupling } \\
\text { interface }\end{array}$ \\
Global FVM & $\begin{array}{l}\text { Continuum } \\
(k=\text { const })\end{array}$ & $\begin{array}{l}\text { Continuum } \\
(k=f(p))\end{array}$ & $\begin{array}{l}\text { Global mesh with } \\
\text { no Mortar }\end{array}$ \\
\end{tabular}

$k$ represents permeability in Table 1 . In Medium $1, k$ is a constant. While in Medium2, $k$ is a function of pressure $k=f(p)$.

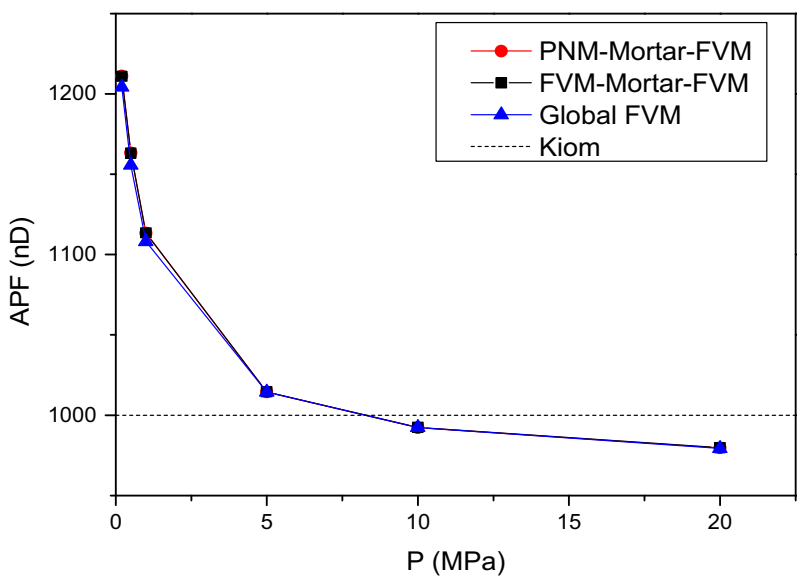

Fig. 5. APF curves of the three conditions.

of the permeabilities calculated by the inlet and outlet of the coupled model is approximately $0.01 \mathrm{nD}$ so that the flow conservation is guaranteed with a minimum computational expense.

\subsubsection{Mesh sizes of the interface}

In this section, the effects of the interface grids on the accuracy and efficiency are discussed. Using the coupled model as Fig. 2, the size of inner pore network is set to $2 \times 2 \times 2 \mu \mathrm{m}^{3}$ and its porosity is set to $10 \%$. According to the pore-size distribution of kerogen (with a mean pore radius of $20 \mathrm{~nm}$, mean throat radius of $14 \mathrm{~nm}$ and standard deviation of lognormal distribution of 0.05 ), the $2 \times 2 \times 2 \mu \mathrm{m}^{3}$ PNM contains approximately $11 \times 11 \times 11$ pores, so there are $11 \times 11$ pores in per interface. With other conditions remain unchanged, two cases of FVM grids and five sets of Mortar grids are used to find the optimal solution. The permeability of inorganic matrix is set to $500 \mathrm{nD}$ and the average pressure is
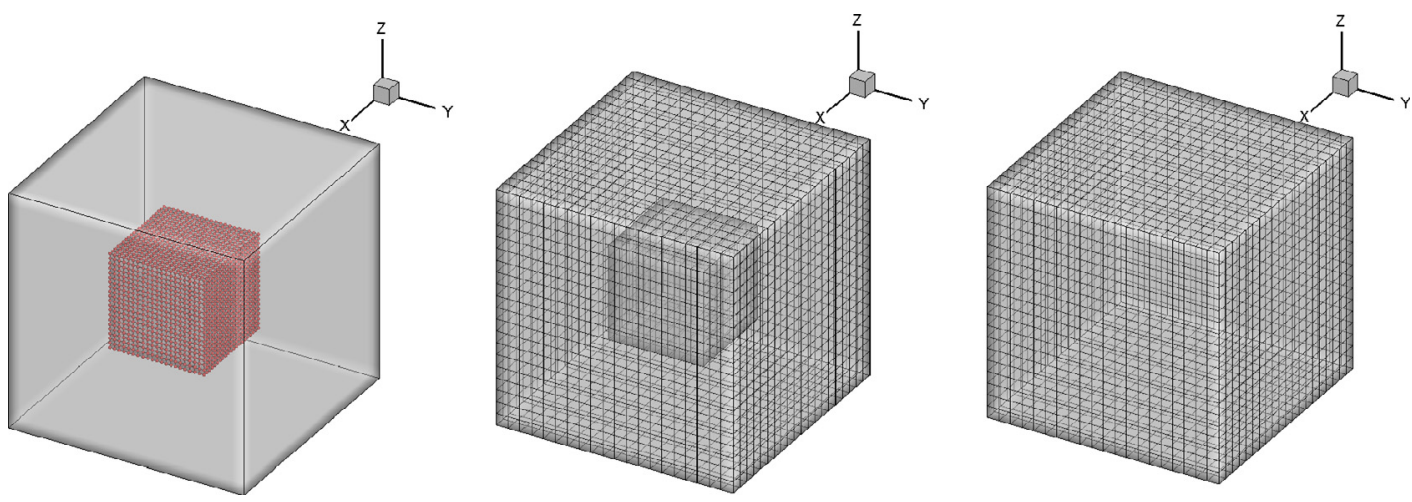

Fig. 4. Images of the three conditions 
Table 2

$K_{c p}$ calculated by different accuracy sets.

\begin{tabular}{|c|c|c|c|c|c|c|}
\hline \multicolumn{3}{|c|}{ Convergence Error } & \multirow[t]{2}{*}{ Computing time (s) } & \multirow[t]{2}{*}{ Inlet $K_{c p}(\mathrm{nD})$} & \multirow[t]{2}{*}{ Outlet $K_{c p}(\mathrm{nD})$} & \multirow[t]{2}{*}{ Deviation $(\mathrm{nD})$} \\
\hline Mortar & PNM & FVM & & & & \\
\hline \multirow[t]{5}{*}{$1 e-6$} & $1 \mathrm{e}-3$ & $1 \mathrm{e}-3$ & 9.3 & 525.85 & 565.11 & 39.26 \\
\hline & $1 e-6$ & $1 \mathrm{e}-6$ & 20.3 & 511.86 & 512.00 & 0.14 \\
\hline & $1 \mathrm{e}-7$ & $1 \mathrm{e}-7$ & 26.6 & 511.93 & 511.94 & 0.01 \\
\hline & $1 \mathrm{e}-8$ & $1 \mathrm{e}-8$ & 34.8 & 511.94 & 511.94 & 0.00 \\
\hline & $1 \mathrm{e}-9$ & $1 \mathrm{e}-9$ & 37.6 & 511.94 & 511.94 & 0.00 \\
\hline
\end{tabular}

Table 3

Calculation parameters and results of different mesh sizes.

\begin{tabular}{|c|c|c|c|c|c|}
\hline Cases & FVM grid at per interface & Mortar grid at per interface & Total number of Mortar nodes & Computation time $(\mathrm{s})$ & $K_{c p}(\mathrm{nD})$ \\
\hline \multirow[t]{5}{*}{ Case1 } & $2 \times 2$ & $1 \times 1$ & 8 & 2.2 & 513 \\
\hline & & $2 \times 2$ & 26 & 6.6 & 512 \\
\hline & & $4 \times 4$ & 98 & 23.7 & 510 \\
\hline & & $8 \times 8$ & 386 & 90.7 & 509 \\
\hline & & $16 \times 16$ & 1538 & - & Singular system \\
\hline \multirow[t]{5}{*}{ Case2 } & $4 \times 4$ & $1 \times 1$ & 8 & 47.8 & 511 \\
\hline & & $2 \times 2$ & 26 & 141.3 & 511 \\
\hline & & $4 \times 4$ & 98 & 517.8 & 511 \\
\hline & & $8 \times 8$ & 386 & 2053.7 & 510 \\
\hline & & $16 \times 16$ & 1538 & - & Singular system \\
\hline
\end{tabular}

$1 \mathrm{MPa}$ here. Calculation parameters and results are shown in Table 3.

In general, a finer Mortar discretization result in a smaller error in flux. However, a $8 \times 8$ Mortar grid results in an ill-conditioned Jacobi matrix and leads to low accuracy. In the extreme case, as the Mortar grid is refined to $16 \times 16$, Jacobi matrix becomes singular, as shown in Table 3 . The condition number of Jacobi matrix and the calculation error will increase when the Mortar grid is excessively refined. Jacobi matrix turns to singular when there is no pores (or finite volume nodes) in one or more Mortar elements. Moreover, the computing expense increases significantly with the refinement of Mortar gird, however, the difference in $K_{c p}$ is less than $1 \%$.

In the previous literature, Balhoff [23] studied the coupling of 826 and 847 pores at the two sides of the interface. For $1 \times 1$, $2 \times 2,4 \times 4,8 \times 8$ Mortar grids, the maximum flux error is only $1.08 \%$ and the maximum pressure error is $0.8 \%$. In Sun's work [30], the sizes of Mortar grid are set to 2, 4, and 20 times the size of adjacent subdomain grids, all those results are shown correct but when it is 20 the computing expense will be greatly saved. Mehmani and Balhoff [31] proposed an Interface Point Partitioning method (IPP) to avoid possible singularity of the interface problem caused by fine Lagrangian Mortars. But in this study, the Jacobi matrix only becomes singular in the extreme case of $16 \times 16$ Mortar grid, with a difference in Kcp by less than $1 \%$. There is a trade-off between accuracy and efficiency, regarding to them simultaneously, the Mortar grid and the inorganic matrix grid in the interface are set to $1 \times 1$ and $4 \times 4$, respectively.

\subsubsection{Parallel scheme}

The solution procedure in Section 2.3 shows that Mortar method is very suitable for parallel computing. Two parallel schemes are proposed here: (1) calculating the components in Jacobi matrix by parallel; (2) calculating the independent subdomains by parallel (Fig. 6). It is found that when the computing complexity of different subdomains varies widely, scheme 1 (Fig. 6b) is easy in parallel design with a small data transfer and a relatively high efficiency. However, when the calculation in each subdomain requires a large amount of storage and those subdomains have a similar computational complexity, using scheme 2 (Fig. 6c) will significantly save computer memory. In this study, there are approximately 1300 pores in a single PNM and 8000 nodes in FVM, scheme 1 is chosen for a faster calculation. But if there are approximately 8000 pores in PNM and 8000 nodes in FVM, using scheme 2 needs a much smaller computer memory.

\section{Sensitivity analysis and discussion}

In this section, the sensitivities of the apparent gas permeability to TOC, kerogen distribution, pore-size distribution and space dimension are investigated. It should be noted that both the regular topology PNMs generated by computer and the real-shale PNMs extracted from FIB/SEM images are analyzed in the sensitivity analysis of kerogen distribution.

\subsection{Calculation parameters design}

In this study, we implemented the sensitivity analysis with the input parameters listed in Table 4 and the state parameters listed in Table 5 . The dimension of the coupled model is set to be $10 \times 10 \times 10 \mu \mathrm{m}^{3}$ with several $2 \times 2 \times 2 \mu \mathrm{m}^{3}$ kerogen blocks. Using the TOC of $3.08 \%$ (volumetric TOC of $8 \%$ ) as an example, the $3 \mathrm{D}$ coupled model is divided into $5 \times 5 \times 5$ blocks and there are 10 kerogen blocks randomly dispersed in it, as shown in Fig. 7.

\subsection{Effects of the total organic content (TOC)}

In order to investigate the effect of TOC on AP, in this section, six cases with different TOC (3.08\%, 4.62\%, 6.15\%, 7.69\%, 9.24\%, 10.78\%) are constructed with the corresponding kerogen block numbers of $10,15,20,25,30,35$. For the $K_{\text {iom }}$ of $500 \mathrm{nD}$ and the pressure boundary condition of $0.2 \pm 0.1 \mathrm{MPa}$, the contour plots of pressure for the six cases are shown in Fig. 8.

A few observations can be made from Fig. 8. First, a continuity of pressure is observed along all of the Mortar interfaces: the interfaces of PNM-PNM and the interfaces of FVM-PNM. Second, the results show that the existence of kerogen indeed affects the gas flow and has a significant impact on the pressure field. In such a low pressure of $0.2 \mathrm{Mpa}$, a significant non-Darcy effect occurs in kerogen so its permeability is much greater than that of inorganic matrix. The interconnected kerogens form a fast pathway for gas transport that results in an extremely small pressure-drop in 


$$
J=\left[\begin{array}{c:c:c:cc}
a_{11} & a_{12} & a_{13} & \cdots & a_{1 n} \\
a_{21} & a_{22} & a_{23} & \cdots & a_{2 n} \\
a_{31} & a_{32} & a_{33} & \cdots & a_{3 n} \\
\vdots \vdots & \vdots & \vdots & \ddots & \vdots \\
a_{n 1} & a_{n 2} & a_{n 3} & \cdots & a_{n n} \\
\mathrm{~A} & \mathrm{~B} & \mathrm{C} & & \\
\hdashline
\end{array}\right.
$$

(a)

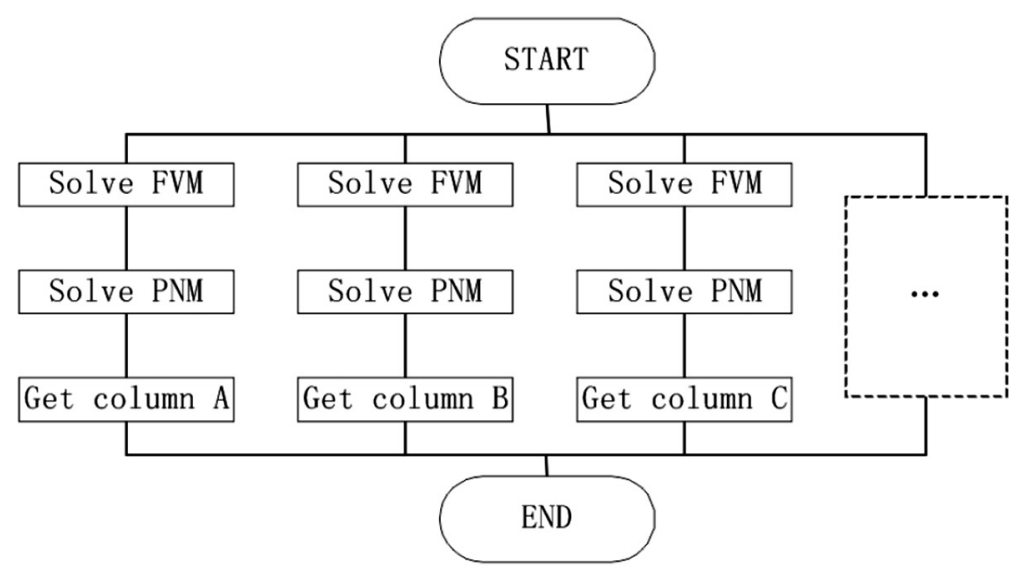

(b)

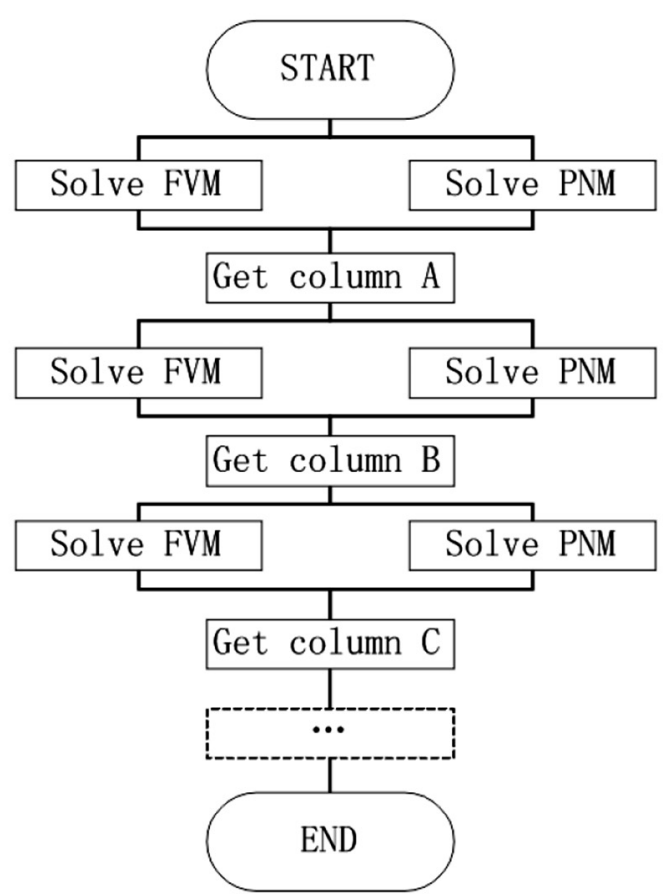

(c)

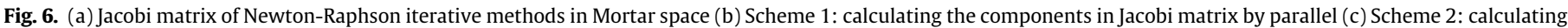
the independent subdomains by parallel. 
Table 4

Base case input parameters.

\begin{tabular}{ll}
\hline Model parameters & Value \\
\hline Coupled model dimension (um) & $10 \times 10 \times 10$ \\
Single kerogen dimension (um) & $2 \times 2 \times 2$ \\
TOC & $3.08 \%, 4.62 \%, 6.15 \%, 7.69 \%, 9.24 \%, 10.78 \%$ \\
Mean pore radius of kerogen (nm) & 20 \\
Mean throat radius of kerogen (nm) & 14 \\
Standard deviations of pores and & $0.05,0.15,0.25,0.35$ \\
$\quad$ throats & $10 \%$ \\
Porosity of kerogen & $11 \times 11 \times 11$ \\
Number of pores in a single kerogen & \\
\hline
\end{tabular}

Table 5

State parameters.

\begin{tabular}{ll}
\hline State parameters & Value \\
\hline Average pressure of the inlet and outlet boundary (Mpa) & $0.2-20$ \\
Permeability of inorganic matrix, $K_{\text {iom }}(\mathrm{nD})$ & $500,1000,5000,1 \mathrm{e} 4$ \\
Gas viscosity, $\mu$ (pa.s) & $1.5 \mathrm{e}-5$ \\
Absolute temperature, $\mathrm{T}(\mathrm{K})$ & 300 \\
Gas compression factor, $\mathrm{Z}$ & 1 \\
\hline
\end{tabular}

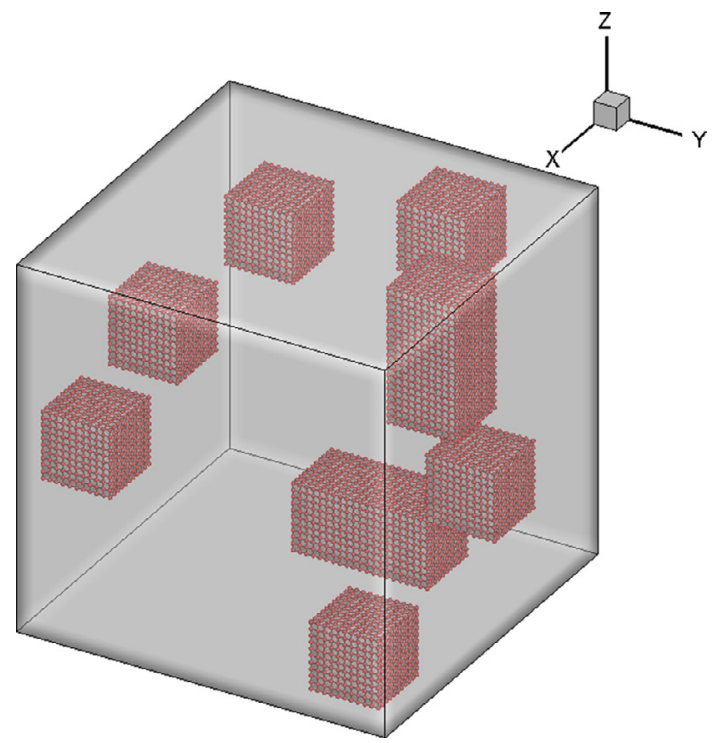

Fig. 7. Example of the coupled model with 10 randomly-dispersed kerogen blocks.

kerogens. It also causes the pressure contours protrude outward near the "kerogen group" and the streamlines converge at both ends of the kerogens. Third, the contours bend more seriously as TOC increases, indicating a stronger heterogeneity.

Fig. 9 shows the APF curves of the coupled model $\left(A P F_{-} M\right)$ with the changing TOC and average pressure at four $K_{\text {iom }}: 500 \mathrm{nD}$, $1000 \mathrm{nD}, 5000 \mathrm{nD}$, and $10,000 \mathrm{nD}$. It indicates that the permeability of shale is much dependent on pressure, and it must be considered as a dynamic reservoir parameter and updated accordingly as the reservoir is being depleted. For comparison, the APF curves of a single kerogen $\left(A P F_{-} K\right)$ and inorganic matrix $\left(K_{\text {iom }}\right)$ are plotted in the same figure. The interesting finding here is that a higher TOC induces a bigger upper-limit of $A P F_{-} M$ but a smaller low-limit of $A P F \_M$. The phenomena can be explained as flows: for the low pressure such as $0.2 \mathrm{Mpa}$, non-Darcy flow plays a dominant role in kerogen causing $A P F \_M$ increases more with a higher TOC; however, for the high pressure such as $20 \mathrm{Mpa}, A P F_{-} K$ is much smaller than $K_{\text {iom }}$ and a higher TOC brings a larger decrease. Fig. 9 also shows the trend that $A P F_{-} M$ becomes closer to $K_{\text {iom }}$ as TOC decreases; on the contrary, $A P F \quad M$ becomes closer to APF $K$ as TOC increases. The deviation between $A P F_{-} M$ and $K_{\text {iom }}$ is less than $10 \%$ with the TOC smaller than $4.62 \%$ and $K_{\text {iom }}$ larger than $1 \mathrm{e} 4 \mathrm{nD}$.

Chen et al. considered that organic matter has nothing to do with the transport channel and gas flow only occurs in inorganic matrix, the mass exchange rate is linearly proportional to the free gas pressure difference between kerogen and inorganic matrix. They supposed the mass exchange is a slow process but the gas flow is a fast process and the two processes are decoupled. It's shown that their assumptions hold under certain conditions. In our model, the coupled gas flow of organic and inorganic is taken into account and mass exchanges occur in the six interfaces of each kerogen block, which fully reflects the contribution of the interconnected pore network of the organic matter to shale permeability. The results show that ignoring the contribution of kerogen to shale permeability will lead to great errors in most of the time, for example, the permeability will be underestimated in the low pressure with an error up to $200 \%$ and overestimated in the high pressure with an error up to $20 \%$. The effect of kerogen on shale permeability is less than $10 \%$ only when the TOC is small than $4.62 \%$ and $K_{\text {iom }}$ is large than $1 \mathrm{e} 4 \mathrm{nD}$. Results show that TOC plays a significant role on shale permeability and agree well with Wang et al.'s study [17].

\subsection{Effects of the kerogen distribution}

In this section, the sensitivities of AP to kerogen distribution in both the regular topology PNMs and real-shale PNMs are studied.

\subsubsection{Regular topology PNMs}

Six random realizations with regular topology PNMs are constructed, and there are 25 kerogen blocks randomly dispersed in them with $\mathrm{TOC}=7.69 \%$, as shown in Fig. 10. The APF curves of the six realizations are shown in Fig. 11.

From Fig. 11, it can be seen that the APF curves have two categories: one category has no intersection of $K_{\text {iom }}$ and $A P F_{-} K$; the other has an intersection and the APF_M curves of different realizations join at the same point. At the intersection point, kerogen distribution has the minimum effect on shale permeability. The impact of kerogen distribution increases as the pressure value moves away from the intersection. The $A P F \_K$ value is enormous with the pressure of less than $0.5 \mathrm{Mpa}$ and the $A P F \_M$ curves of different distributions are quite different. $A P F_{-} K$ gets close to $K_{\text {iom }}$ when the pressure approaches the intersection points, which causes the coupled model to be a homogeneous body and the $A P F \_M$ curves of different kerogen distributions to join. When the pressure is greater than $10 \mathrm{Mpa}$, gas flow in kerogen tends to be Darcy's flow which leads to an approximately level $A P F \_M$ curve and a small permeability difference of the kerogen distributions.

To better quantify the effects of kerogen distribution, the relative deviation between the maximum and minimum permeability of three stochastic realizations at different TOC levels are calculated, as shown in Table 6.

Table 6 shows that: (1) in general, the impact of kerogen distribution increases as TOC increases; (2) the relative deviation has a minimum value because the coupled model tends to a homogeneous body when the pressure approaches the intersection points; (3) When TOC is less than $11 \%$ and the pressure is greater than $0.1 \mathrm{Mpa}$, the permeability deviation of different kerogen distributions is less than $10 \%$ and thus, kerogen distribution has a negligible influence on shale permeability. The results is qualitatively consistent with Wang's conclusion [17].

\subsubsection{Real-shale PNMs}

The discussions above base on regular networks generated by computer. However, for a real shale, irregular pore network is essential, so a real shale model generated from FIB/SEM images 

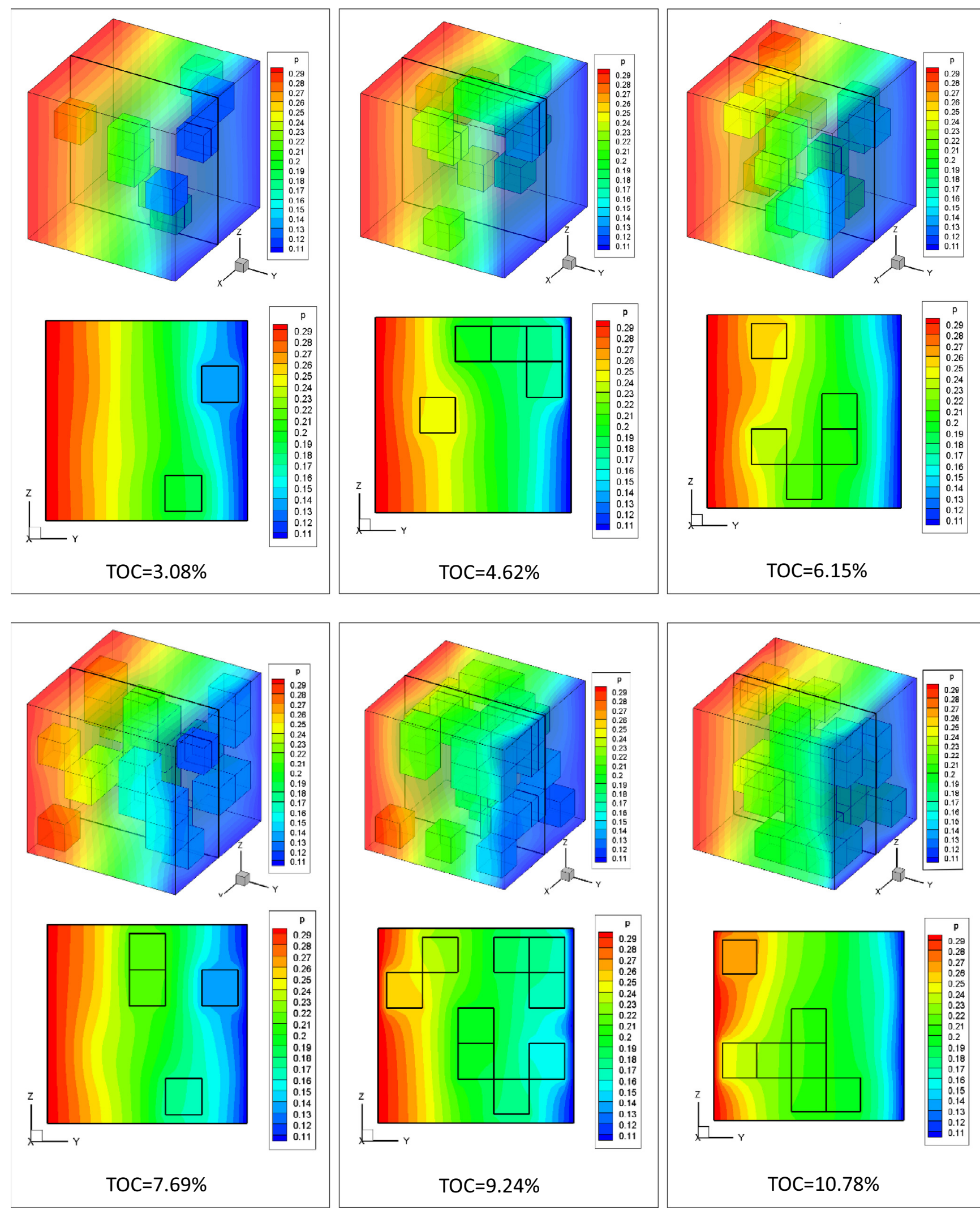

TOC $=7.69 \%$

Fig. 8. Contour plots of pressure for the six cases with TOC varies from $3.08 \%$ to $10.78 \%$. The pressure fields of the center section of $\mathrm{x}$-direction are also shown for clarity.

is discussed in this section. The data of grey black mud shale from Longmaxi formation, Sichuan, China is adopted here. This shale belongs to marine shale, and the buried depth is approximately 1319.2 meters, with Ro of 2.3 and the TOC of 3.6\% (Fig. 12a). The voxel numbers of the entire dataset is $1024 \times 884 \times 406$, and the voxel size is $10 \mathrm{~nm}$. The light grey is inorganic matrix, which is mainly composed of quartz and clay minerals. The dark grey represents kerogen and the darkest parts in it are void. It can be 

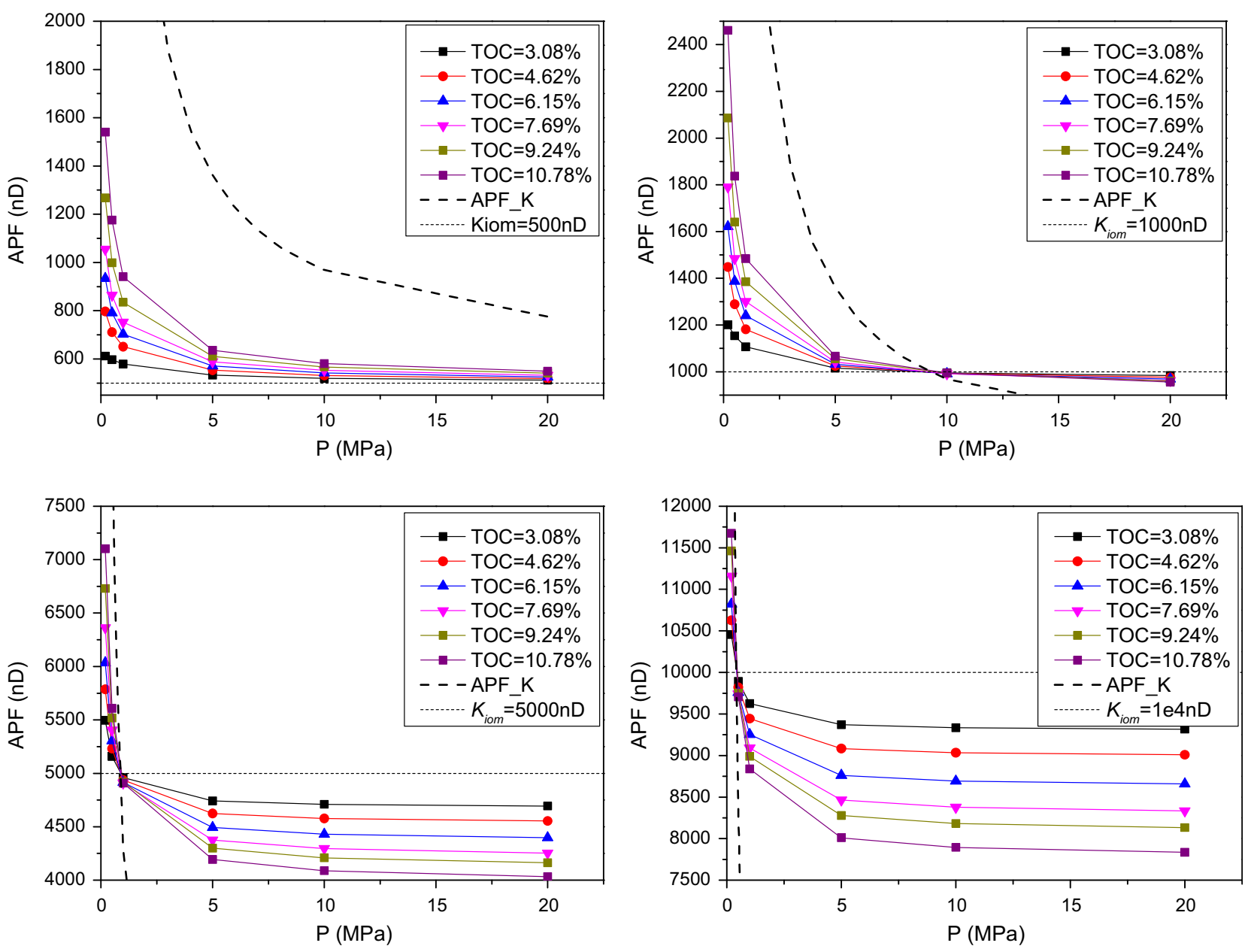

Fig. 9. APF curves of the six cases with TOC varies from $3.08 \%$ to $10.78 \%$ at four $K_{\text {iom }}$.
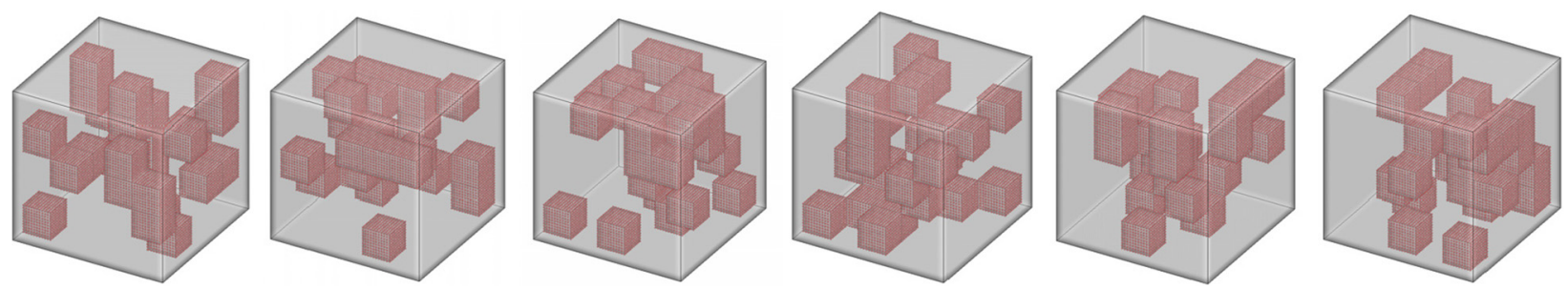

Fig. 10. Six realizations with random kerogen distributions (r1-6).

observed that kerogens indeed disperse in the inorganic matrix. In this study, the $A B$ (axis \& ball) algorithm [26,32] is employed to extract the kerogen blocks and get the corresponding PNMs, those PNMs are coupled to the inorganic matrix and subsequently the real-shale model is found. For the real shale in Fig. 12a, seven PNMs (S1, S2, S3, S4, S5, S6, S7) are obtained (Fig. 13), and the real-shale coupled model is established, as shown in Fig. 12b.

Flowing porosity is the ratio of volume of throats with flux magnitude larger than 0 to total volume. $\mathrm{Np}$ and $\mathrm{Nt}$ are the numbers of pores and throats in the pore network, respectively. CNavg is the average coordination number. Ravg is the arithmetic mean throat radius. For more details about $A B$ extraction algorithm and the properties of those kerogens, one can refer to Jiang et al.'s work [26].

The TOC and pore-size distributions are approximately constant in the real shale within a certain formation but the kerogen distri- bution is of considerable uncertainty. Therefore, the effect of kerogen distribution is discussed here. We calculated the permeability in the $\mathrm{x}$ - and $\mathrm{y}$-direction and found that there is a large difference between different directions due to the strong anisotropy in shale. Therefore, the two directions are separated in the analysis of the influences of kerogen distribution, with kerogens re-randomized by only translating but not rotating. Fig. 14 shows the four realizations of random kerogen distributions of the real shale model LMX in Fig. 12b. Assuming the inorganic matrix permeability is $1000 \mathrm{nD}$, the APF curves in $\mathrm{x}$ direction and $\mathrm{y}$ direction of those realizations are shown in Fig. 15.

As can be seen from Fig. 15, the anisotropy and heterogeneity of pore network are significant in the real shale which causes the permeabilities vary in different directions. However, kerogen distributions have a negligible influence on the permeability of real shale with a maximum difference of $3 \%$. 

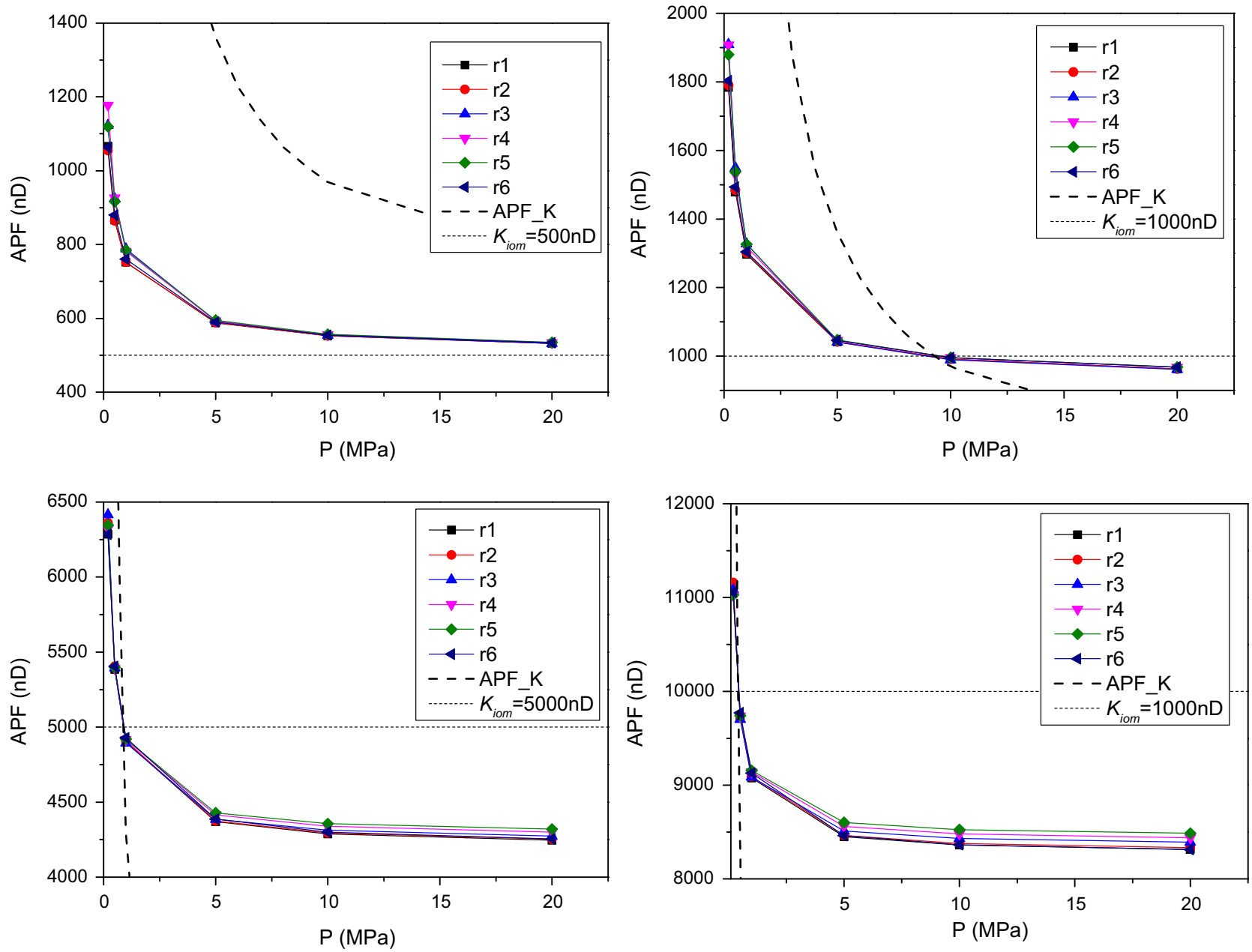

Fig. 11. APF curves of different kerogen-distribution realizations at four $K_{\text {iom }}$.

Table 6

Deviation statistics of different kerogen distributions.

\begin{tabular}{|c|c|c|c|c|c|c|}
\hline \multirow[t]{2}{*}{ TOC } & \multicolumn{6}{|c|}{ Boundary Pressure condition (Mpa) } \\
\hline & $0.2 \pm 0.1$ & $0.5 \pm 0.1$ & $1 \pm 0.1$ & $5 \pm 0.1$ & $10 \pm 0.1$ & $20 \pm 0.1$ \\
\hline $3.08 \%$ & $2.40 \%$ & $1.46 \%$ & $0.75 \%$ & $0.02 \%$ & $0.02 \%$ & $0.02 \%$ \\
\hline $4.62 \%$ & $4.90 \%$ & $2.37 \%$ & $1.18 \%$ & $0.03 \%$ & $0.03 \%$ & $0.03 \%$ \\
\hline $6.15 \%$ & $5.60 \%$ & $2.97 \%$ & $1.44 \%$ & $0.04 \%$ & $0.05 \%$ & $0.07 \%$ \\
\hline $7.69 \%$ & $7.04 \%$ & $4.79 \%$ & $2.35 \%$ & $0.08 \%$ & $0.13 \%$ & $0.16 \%$ \\
\hline $9.24 \%$ & $9.70 \%$ & $4.37 \%$ & $1.83 \%$ & $0.08 \%$ & $0.13 \%$ & $0.15 \%$ \\
\hline $10.78 \%$ & $15.32 \%$ & $7.90 \%$ & $3.66 \%$ & $0.11 \%$ & $0.15 \%$ & $0.16 \%$ \\
\hline
\end{tabular}

\subsection{Effects of pore size distribution}

In this section, the sensitivity of AP to pore-size distribution is presented. The mean pore radius is set to a constant as Table 4 but the standard deviations $\sigma$ of lognormal pore radius distribution range from 0.05 to 0.45 , as listed in Table $8 . \sigma$ reflects the degree of dispersion of pore size and it actually represents the heterogeneity of the PNMs. Thus, the effect of heterogeneity within kerogens on the permeability of the coupled model is analyzed.

Fig. 16 illustrates the APF curves of different $\sigma$. The result shows that the increase in $\sigma$ increases the permeability of the coupled model. The intersection point of $K_{\text {iom }}$ and $A P F \_M$ moves right when $\sigma$ increases. When $\sigma$ is big enough $(>0.25)$, the Darcy's permeabilities of those kerogens are greater than that of the inorganic matrix so that the APF_M curve and $K_{\text {iom }}$ curve have no intersection. From
Fig. 17, it can be seen that the increase of the permeability of the coupled model is non-linear with increasing $\sigma$. The maximum deviation of different $\sigma$ is $17 \%$. The results show that using the mean value of pore radius is not sufficient and the pore size distribution should be taken into consideration when calculating the permeability of kerogen.

In addition, in order to examine whether the results in Section 3.3 are universal, three random realizations of kerogen distribution are calculated with $\sigma$ ranged from 0.05 to 0.45 , as shown in Table 9, and the maximum relative deviations of AP are also given.

Table 9 depicts that a larger $\sigma$ induces a greater relative deviation of different kerogen distributions. This is because the range of the kerogen pore radius spans wide and the heterogeneity inside the kerogen becomes strong as $\sigma$ increases, and then those strong heterogeneous kerogens disperse randomly in the coupled model 


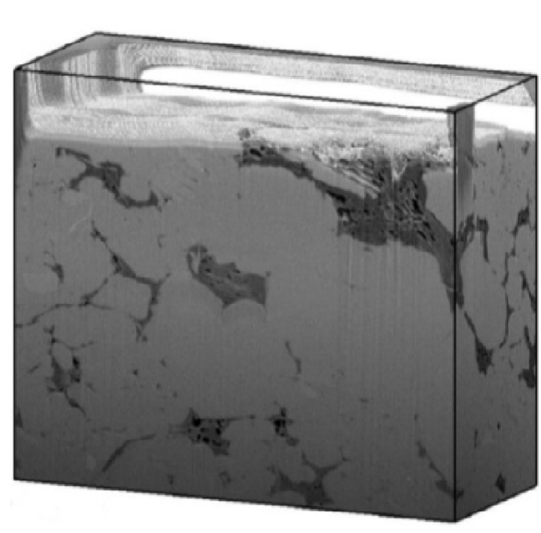

(a)

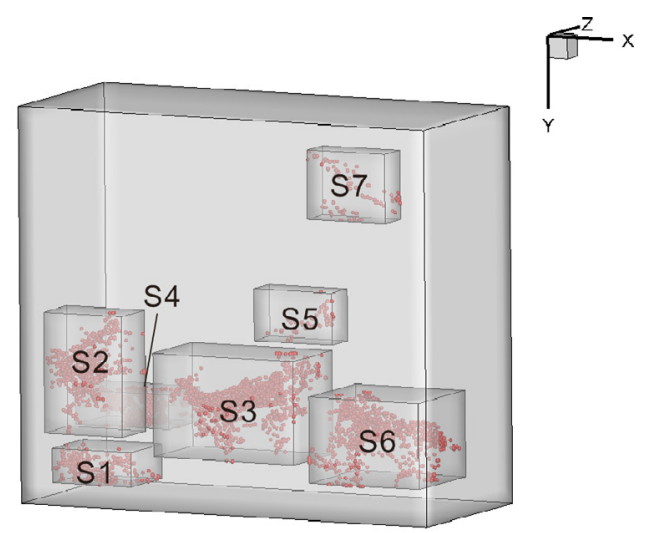

(b)

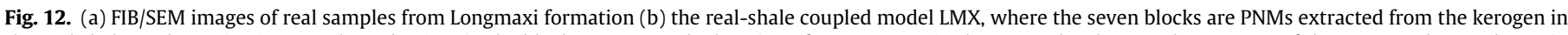

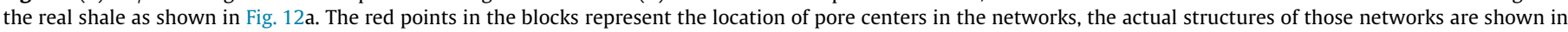
Fig. 13 and their parameters from $A B$ model are shown in Table 7.

Table 7

Parameters obtained by AB model.

\begin{tabular}{|c|c|c|c|c|c|c|}
\hline Kerogen Sample & Flowing Porosity & $\mathrm{Np}$ & $\mathrm{Nt}$ & $\mathrm{CN}_{\mathrm{avg}}$ & $\mathrm{R}_{\mathrm{avg}}(\mathrm{nm})$ & Tortuosity \\
\hline S1 & $0.67 \%$ & 154 & 298 & 2.45 & 11.08 & 1.62 \\
\hline S2 & $0.24 \%$ & 380 & 913 & 2.44 & 9.00 & 2.47 \\
\hline S3 & $0.25 \%$ & 873 & 2235 & 2.77 & 9.12 & 1.65 \\
\hline S4 & $0.27 \%$ & 410 & 892 & 2.60 & 8.57 & 2.05 \\
\hline S5 & $1.61 \%$ & 25 & 30 & 1.44 & 10.18 & 1.32 \\
\hline S6 & $0.26 \%$ & 586 & 1542 & 2.68 & 10.11 & 1.99 \\
\hline S7 & $0.15 \%$ & 41 & 62 & 1.93 & 13.42 & 1.42 \\
\hline
\end{tabular}

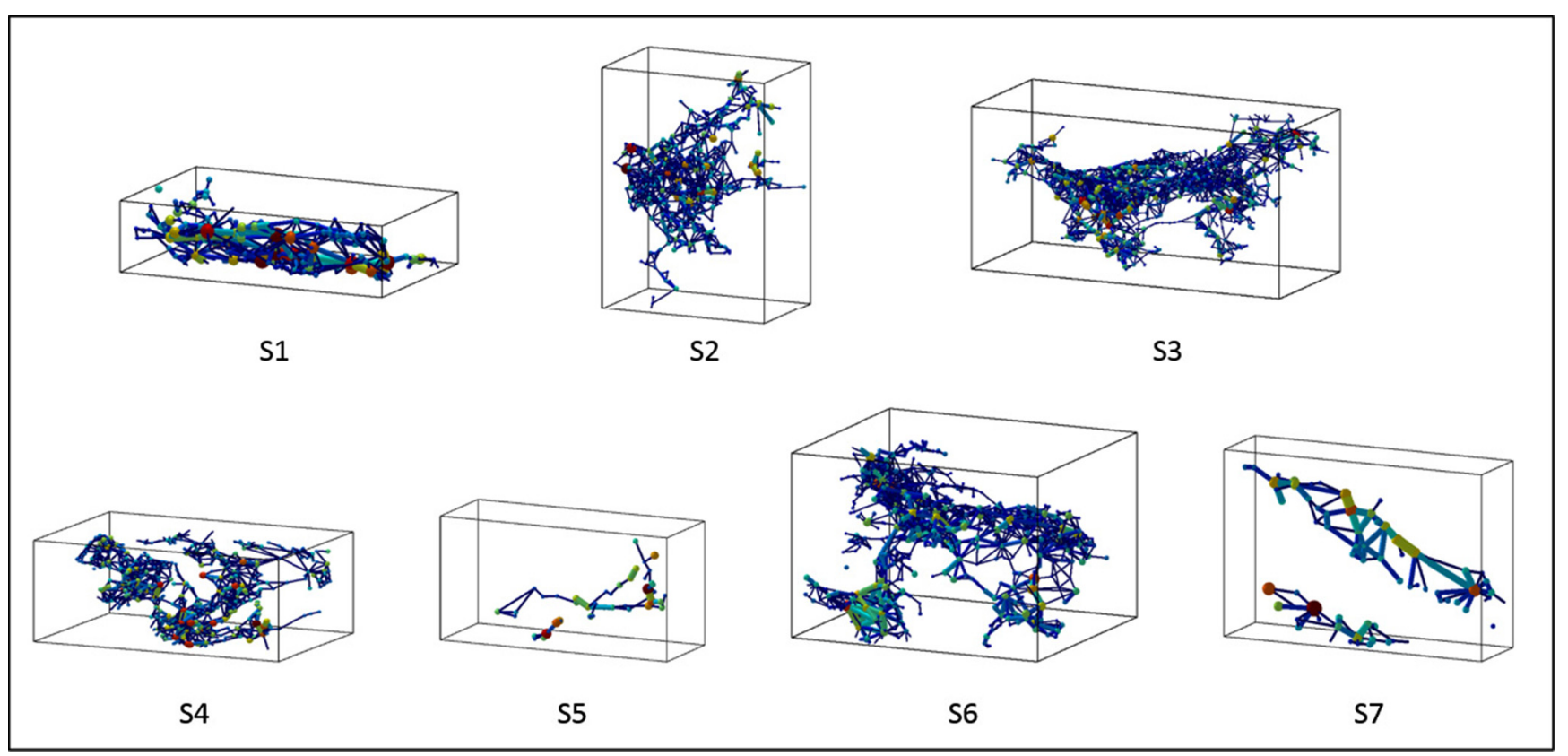

Fig. 13. Structures of the PNMs in Fig. $12 \mathrm{~b}$.

which increases the uncertainty of shale permeability. However, the relative deviations of kerogen distributions still are within $8.44 \%$ even in an extremely large $\sigma$ (such as 0.45 ) and an extremely low pressure (such as $0.2 \mathrm{Mpa}$ ). Consequently, the kerogen distribution has a negligible effects on AP in different pore-size distributions and the conclusions in Section 3.3 are extended.

\subsection{Effects of the dimension}

\subsubsection{Comparisons of $2 D$ and $3 D$ model}

Since the 3D models of shale have a considerable computing, 2D models are widely adopt in the recent published works. In order to analyze the effect of model dimension, coupled models in both 2D 

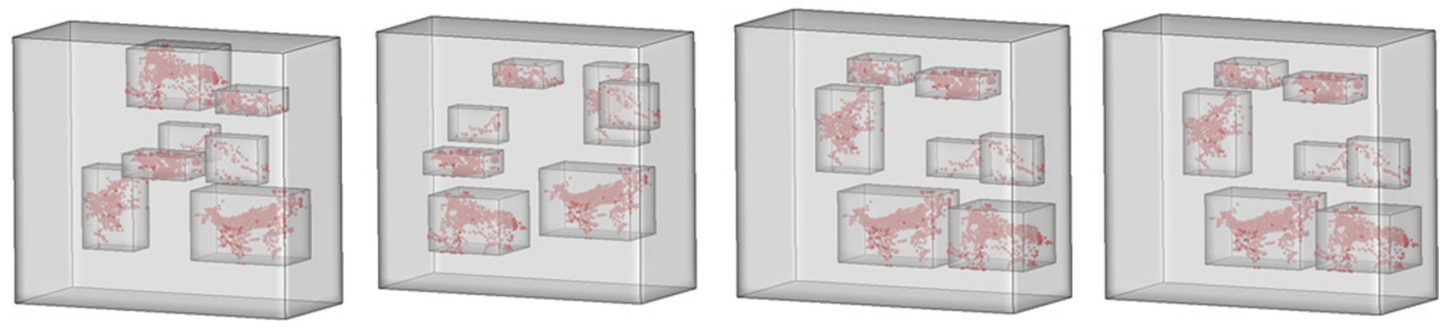

Fig. 14. Realizations of random kerogen distribution (LMXr1-r4), they are the same with the real-shale model LMX in Fig. 12b except kerogen distribution.
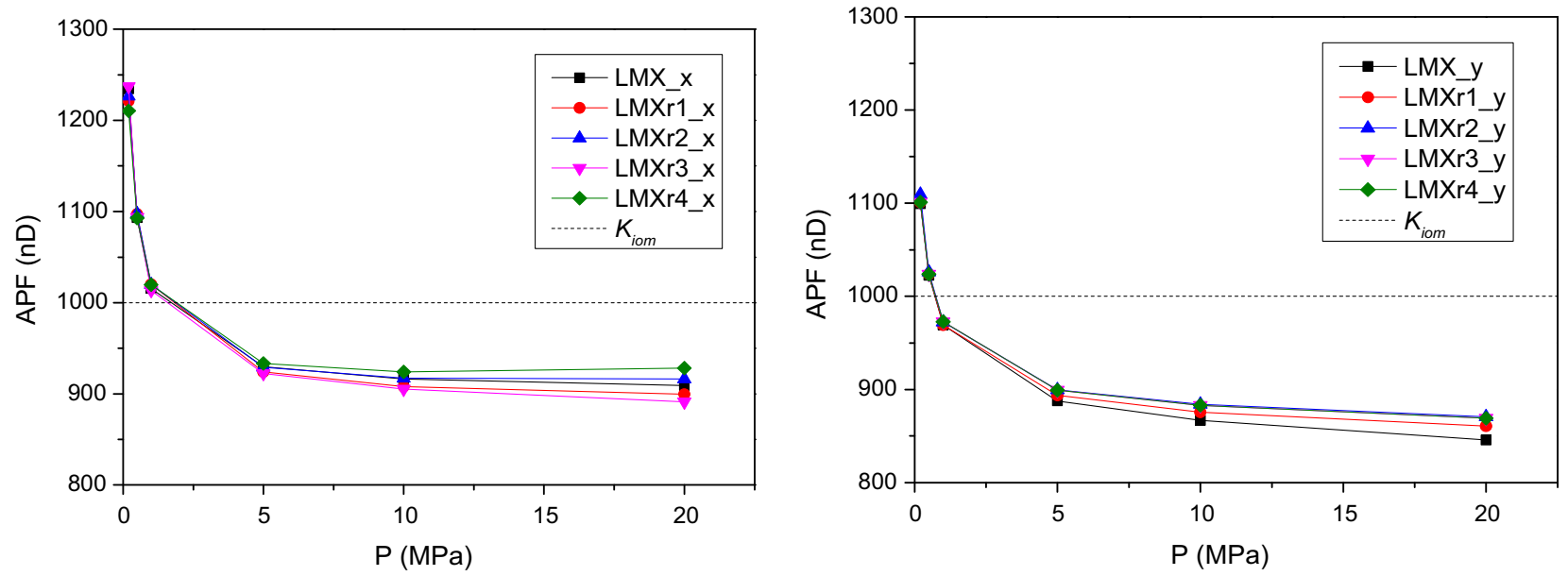

Fig. 15. APF curves of different kerogen distributions in both $\mathrm{x}$ and $\mathrm{y}$ direction.

Table 8

Parameters of the four pore-size distributions.

\begin{tabular}{|c|c|c|c|c|}
\hline$\sigma$ & MAX pore radius(nm) & MIN pore radius(nm) & MAX throat radius(nm) & MIN throat radius(nm) \\
\hline 0.05 & 23.2 & 17.0 & 16.7 & 11.5 \\
\hline 0.15 & 35.1 & 12.1 & 24.2 & 7.6 \\
\hline 0.25 & 44.2 & 9.0 & 34.5 & 5.4 \\
\hline 0.35 & 59.6 & 6.4 & 49.2 & 4.4 \\
\hline 0.45 & 96.2 & 3.9 & 65.2 & 2.9 \\
\hline
\end{tabular}

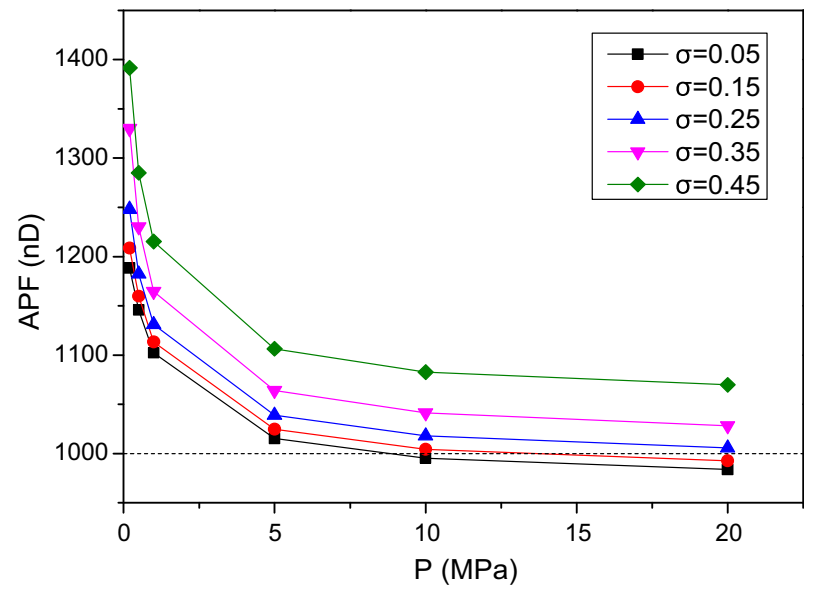

Fig. 16. APF curves of different $\sigma$.

and 3D are constructed. The 2D model is designed with the same TOC and porosity as the 3D model (Fig. 18). Here, the TOC and kerogen porosity are set to $7.69 \%$ and $10 \%$, so the porosity of the coupled model is also the same. The 3D model has 25 kerogen

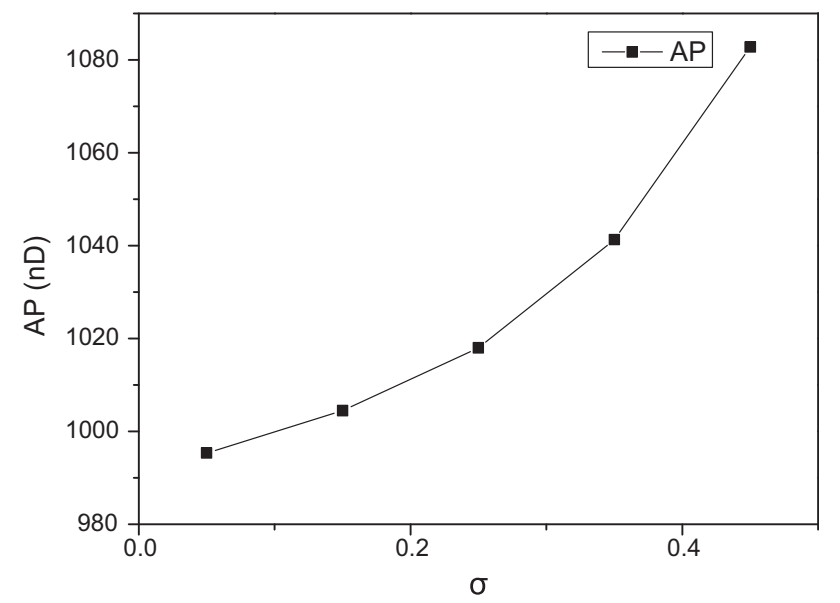

Fig. 17. Variation of apparent permeability with $\sigma$. blocks, and each $2 \times 2 \times 2 \mu \mathrm{m}^{3}$ block contains $11 \times 11 \times 11$ pores and the corresponding 2D model contains 5 PNMs and the model thickness is $2 / 11 \mu \mathrm{m}$. 
Table 9

Maximum relative deviations of kerogen distributions in different $\sigma$.

\begin{tabular}{|c|c|c|c|c|c|c|}
\hline \multirow[t]{2}{*}{$\sigma$} & \multicolumn{6}{|c|}{ Boundary Pressure condition (Mpa) } \\
\hline & $0.2 \pm 0.1$ & $0.5 \pm 0.1$ & $1 \pm 0.1$ & $5 \pm 0.1$ & $10 \pm 0.1$ & $20 \pm 0.1$ \\
\hline 0.05 & $2.40 \%$ & $1.46 \%$ & $0.75 \%$ & $0.02 \%$ & $0.02 \%$ & $0.02 \%$ \\
\hline 0.15 & $5.25 \%$ & $2.84 \%$ & $1.38 \%$ & $0.06 \%$ & $0.02 \%$ & $0.02 \%$ \\
\hline 0.25 & $5.68 \%$ & $2.86 \%$ & $1.43 \%$ & $0.13 \%$ & $0.03 \%$ & $0.01 \%$ \\
\hline 0.35 & $6.59 \%$ & $3.82 \%$ & $1.97 \%$ & $0.14 \%$ & $0.04 \%$ & $0.02 \%$ \\
\hline 0.45 & $8.44 \%$ & $5.01 \%$ & $3.13 \%$ & $0.92 \%$ & $0.53 \%$ & $0.23 \%$ \\
\hline
\end{tabular}
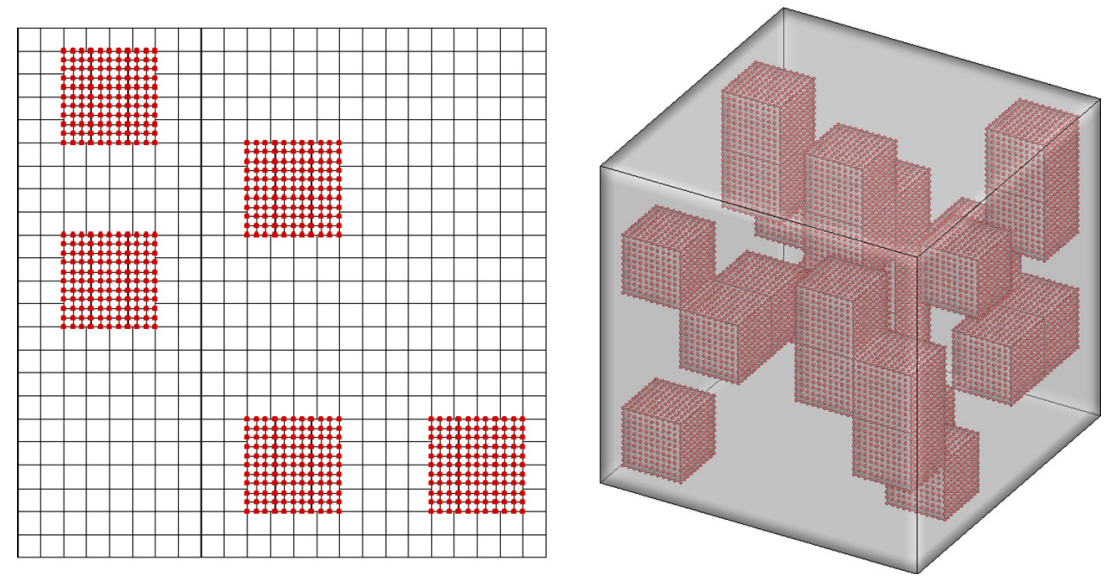

Fig. 18. 2D and 3D model with the same TOC and porosity.

The $K_{i o m}$ is set to 500 nd and the average pressure varies from 0.2 to $20 \mathrm{Mpa}$. And then the permeability of the $2 \mathrm{D}\left(K_{2 D}\right)$ and 3D model $\left(K_{3 D}\right)$ are calculated. The results are normalized using the ratio of $K_{2 D}$ to $K_{3 D}$, as shown in Fig. 19.

Fig. 19 shows that, the deviation of the 2D and 3D model tends to be steady by approximately $4 \%$ when the pressure is greater than $10 \mathrm{Mpa}$, as gas flow tends to be Darcy's flow in kerogens. The decrease in pressure increases the deviation of the 2D and $3 \mathrm{D}$ model, with a maximum error of $37 \%$. However, the computing time of 2D model and 3D model are 3s and 1800s. So the efficiency of $2 \mathrm{D}$ model is approximately 600 times that of 3D model. It is concluded that when the pressure is larger than $10 \mathrm{Mpa}$, the error of the $2 \mathrm{D}$ model is approximately $4 \%$, accompany with a significantly high efficiency.

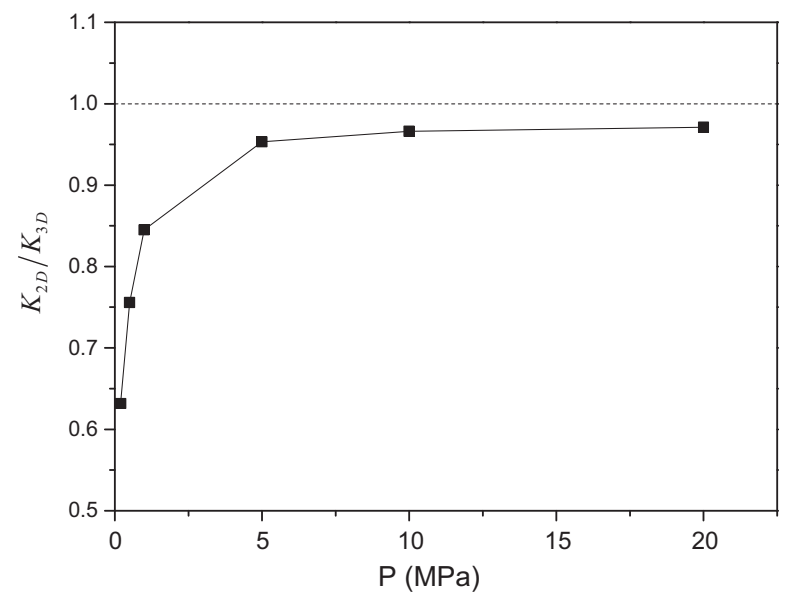

Fig. 19. Ratio of the permeability calculated by $2 \mathrm{D}$ model and 3D model.

\subsubsection{Quantitative comparisons with other results}

2D model is widely used in the published works so far. In order to conduct quantitative comparisons with other results, we build a 2D model to keep the parameters (sample size, gas type, porosity, TOC, Temperature, pressure and pore size et al.) consistent with Naraghi and Javadpour's work [16], as shown in Table 10.

We build four realizations with random kerogen distributions and calculate their permeabilties. In organic kerogen, PNM is utilized to represent it with a fixed pore size distribution. In inorganic matrix, FVM is used to represent it and its permeability is calculated by $K=\frac{\phi \bar{r}^{2}}{8 \tau}$, where $\phi$ and $\tau$ are the porosity and tortuosity, $\bar{r}$ is the mean pore radius. The mean values and standard deviations of those realizations are shown in Table 11, compared with the results of Naraghi and Javadpour's.

Table 11 shows that the permeability calculated by the Mortar coupled model is in good agreement with Naraghi and Javadpour's. The standard deviation is smaller than that of Naraghi and Javadpour as the mean pore radius is employed in the inorganic matrix so it is more homogeneous in this study. Although the 2D model is calculated here, in the previous discussion it has been presented

Table 10

Input parameters for quantitative comparisonss.

\begin{tabular}{ll}
\hline Input parameters & $\begin{array}{l}\text { Data reported by Naraghi \& Javadpour } \\
(2015)[16]\end{array}$ \\
\hline Sample size & $10 \mu \mathrm{m} \times 10 \mu \mathrm{m}$ \\
Gas type & Methane, $16.04 \mathrm{~g} / \mathrm{mol}$ \\
Porosity & $10 \%$ \\
Volumetric TOC & $12 \%$ \\
Temperature & $300 \mathrm{~K}$ \\
Average pressure & $10 \mathrm{Mpa}$ \\
Tortuosity & 2 \\
Organic matter pore-size & $\mu=0.4, \sigma=0.18$ \\
$\quad$ distribution & $25 \mathrm{~nm}$ \\
Inorganic matter average pore & \\
$\quad$ diameter &
\end{tabular}


Table 11

Results comparision.

\begin{tabular}{lll}
\hline & Mean Permeability & Standard Deviation \\
\hline Naraghi \& Javadpour(2015) [16] & $0.70 \mu \mathrm{D}$ & 0.23 \\
This work & $0.72 \mu \mathrm{D}$ & 0.04 \\
\hline
\end{tabular}

that the deviation of the 2D and 3D model is approximately $4 \%$ in the pressure of $10 \mathrm{Mpa}$.

\section{Conclusion}

In this study, a newly developed model coupling kerogen and inorganic matrix is proposed to calculate the micro-scale permeability of shale. Considering the different flow characteristics in kerogen and inorganic matrix, they are represented by PNM and FVM respectively, and Mortar method is employed to ensure the pressure continuity and the weak match of fluxes at their interfaces. The computing efficiency of Mortar method and the influence of different sensitive parameters on shale permeability are investigated. Following are the conclusions drawn from the study:

- When the grids of FVM and Mortar space are set to $4 \times 4$ and $1 \times 1$ at per interface, the accuracy is guaranteed with a minimum computing expense.

- When the computing complexity of different subdomains varies widely (for example, there are approximately 1300 pores in a single PNM and 8000 nodes in FVM), calculating the components in Jacobian matrix by parallel has a higher efficiency.

- Ignoring the contribution of kerogen to shale permeability will lead to great errors in most cases, for example, the permeability will be underestimated in low pressure with an error up to $200 \%$ and overestimated in high pressure with an error up to $20 \%$. The effect of kerogen on shale permeability is less than $10 \%$ only when TOC is smaller than $5 \%$ and inorganic matrix permeability is larger than $10,000 \mathrm{nD}$.

- When TOC is less than $11 \%$ and the pressure is higher than $0.1 \mathrm{MPa}$, kerogen distribution has a negligible influence on shale permeability with the deviation of less than $10 \%$.

- The permeability of the coupled model increases nonlinearly with increasing standard deviations of lognormal distribution of pore and throat, $\sigma$.

- When the pressure is larger than $10 \mathrm{MPa}$, the deviation between the permeability calculated by 2D and 3D model is approximately $4 \%$.

This study finds that kerogen distribution has limited effect on shale permeability, suggesting the feasibility of establishing an equivalent model. For a real shale, different blocks of kerogen can be integrated and thus, the TOC, pore size distribution and anisotropy will be reflected in the equivalent kerogen block for a larger scale simulations. The 3D couple model proposed here affords a foundation for further upscaling of shale permeability to REV scale and reservoir scale.

\section{Acknowledgments}

This work is supported by the Strategic Priority Research Program of the Chinese Academy of Sciences (Grant No. XDB10020302), the National Natural Science Foundation of China (Grant No. 41690132), the Major National Science and Technology Special Program of China (Grant No. 2017ZX05037-001), the National Natural Science Foundation of China (Grant No. 41574129) and the National Program on Key Basic Research Project (973 Program, Grant No. 2014CB239004).

\section{References}

[1] Fan D, Ettehadtavakkol A. Semi-analytical modeling of shale gas flow through fractal induced fracture networks with microseismic data. Fuel 2017; 193:444-59.

[2] Chen L, Kang Q, Dai Z, Viswanathan HS, Tao W. Permeability prediction of shale matrix reconstructed using the elementary building block model. Fuel 2015;160:346-56.

[3] Ma Y, Pan Z, Zhong N, Connell LD, Down DI, Lin W, et al. Experimental study of anisotropic gas permeability and its relationship with fracture structure of Longmaxi Shales, Sichuan Basin, China. Fuel 2016;180:106-15.

[4] Singh H, Javadpour F. Langmuir slip-Langmuir sorption permeability model of shale. Fuel 2016;164:28-37.

[5] Cao P, Liu J, Leong Y-K. A fully coupled multiscale shale deformation-gas transport model for the evaluation of shale gas extraction. Fuel 2016;178:103-17.

[6] Jiang J, Younis RM. A multimechanistic multicontinuum model for simulating shale gas reservoir with complex fractured system. Fuel 2015;161:333-44.

[7] Loucks RG, Reed RM, Ruppel SC, Jarvie DM. Morphology, genesis, and distribution of nanometer-scale pores in siliceous mudstones of the Mississippian Barnett Shale. J Sedimentary Res 2009;79:848-61.

[8] Ambrose RJ, Hartman RC, Diaz-Campos M, Akkutlu IY, Sondergeld CH. Shale gas-in-place calculations part I: new pore-scale considerations. Spe J 2012;17:219-29.

[9] Sondergeld CH, Ambrose RJ, Rai CS, Moncrieff J. MicroStructural studies of gas shales. Pittsburgh: Society of Petroleum Engineers; 2010.

[10] Fathi E, Akkutlu IY. Lattice boltzmann method for simulation of shale gas transport in kerogen. Spe J 2011;18:27-37.

[11] Akkutlu IY, Fathi E. Multiscale gas transport in shales with local kerogen heterogeneities. Spe J 2012;17:1002-11.

[12] Akkutlu IY, Efendiev Y, Savatorova V. Multi-scale asymptotic analysis of gas transport in shale matrix. Transp Porous Media 2015;107:235-60.

[13] Akkutlu IY, Efendiev Y, Vasilyeva M. Multiscale model reduction for shale gas transport in fractured media. Comput Geosci 2015;1:1-21.

[14] Talonov A, Vasilyeva M. On numerical homogenization of shale gas transport. J Comput Appl Math 2016;301:44-52.

[15] Song W, Yao J, Li Y, Sun H, Zhang L, Yang Y, et al. Apparent gas permeability in an organic-rich shale reservoir. Fuel 2016;181:973-84.

[16] Naraghi ME, Javadpour F. A stochastic permeability model for the shale-gas systems. Int J Coal Geol 2015;140:111-24.

[17] Wang J, Chen L, Kang O, Rahman SS. Permeability prediction of organic shale with generalized lattice Boltzmann model considering surface diffusion effect. Fuel 2016;181:478-90.

[18] Chen C. Multiscale imaging, modeling, and principal component analysis of gas transport in shale reservoirs. Fuel 2016:182:761-70.

[19] Battiato I, Tartakovsky DM, Tartakovsky AM, Scheibe TD. Hybrid models of reactive transport in porous and fractured media. Adv Water Resour 2011;34:1140-50.

[20] Sheng Q, Thompson K. Dynamic coupling of pore-scale and reservoir-scale models for multiphase flow. Water Resour Res 2013;49:5973-88.

[21] A. Mehmanic, J.H. Chen, M. Prodanovic, C. Edwards, A Pore Scale Analysis of Restricted Diffusion in Shale Gas Media, SPE/AAPG/SEG Unconventional Resources Technology Conference, Society of Petroleum Engineers, 2014.

[22] Tomlin AS, Ghorai S, Hart G, Berzins M. 3-D Multi-scale air pollution modelling using adaptive unstructured meshes. Environ Model Software 2000:15:681-92.

[23] Balhoff MT, Thomas SG, Wheeler MF. Mortar coupling and upscaling of porescale models. Comput Geosci 2008;12:15-27.

[24] Jerauld GR, Salter SJ. The effect of pore-structure on hysteresis in relative permeability and capillary pressure: pore-level modeling. Transp Porous Media 1990;5:103-51.

[25] Idowu NA, Blunt MJ. Pore-scale modelling of rate effects in waterflooding. Transp Porous Media 2010;83:151-69.

[26] Jiang W, Lin M, Yi Z, Li H, Wu S. Parameter determination using 3D FIB-SEM images for development of effective model of shale gas flow in nanoscale pore clusters. Transp Porous Media 2017;117:5-25.

[27] Javadpour F. Nanopores and apparent permeability of gas flow in mudrocks (shales and siltstone). J Can Pet Technol 2009;48:16-21.

[28] C. Bernardi, A new nonconforming approach to domain decomposition: the Mortar element method, Nonliner Partial Differential Equations \& Their Applications 1994; pp: 13-51.

[29] Balhoff MT, Thompson KE, Hjortsø M. Coupling pore-scale networks to continuum-scale models of porous media. Comput Geosci 2007;33. 393-10.

[30] Sun T. Upscaling and multiscale simulation by bridging pore scale and continuum scale models, in. Austin: The University of Texas at Austin; 2012. p. 150.

[31] Mehmani Y, Balhoff MT. Bridging from pore to continuum: a hybrid mortar domain decomposition framework for subsurface flow and transport. Siam J Multiscale Model Simulation 2014;12:667-93.

[32] Yi ZX, Lin M, Jiang WB, Zhang ZB, Li HS, Gao J. Pore network extraction from pore space images of various porous media systems (accepted article). Water Resour Res 2017 